\title{
Effects of target set size on feelings of knowing and cued recall: Implications for the cue effectiveness and partial-retrieval hypotheses
}

\author{
THOMAS A. SCHREIBER \\ University of Kansas, Lawrence, Kansas
}

\begin{abstract}
Target set size refers to the number of preexisting connections a studied word has to closely related concepts in long-term memory. The purpose of this investigation was to determine whether target set size influences feeling-of-knowing (FOK) ratings. The results showed that ratings were higher for targets connected to smaller sets, as compared with those connected to larger sets. Comparable effects were obtained with the use of different encoding strategies, including concreteness classifications and vowel naming, and with both meaningfully and phonologically related test cues. These findings indicate that FOKs are sensitive to competition between concepts linked to the target and that this sensitivity is independent of encoding strategy and type of test cue. Response time measures indicated that FOKs are sensitive to competition that arises when concepts are activated in parallel, whereas recall is more sensitive to competition that arises during sampling associated with search. Implications for various cue-based and target-based explanations of FOK effects are discussed.
\end{abstract}

When people are unable to remember previously learned information they nevertheless can have a sense of whether they will be able to remember it later on (see, e.g., Blake, 1973; Eysenck, 1979; Hart, 1967; Leonesio \& T. O. Nelson, 1990; Metcalfe, 1993; T. O. Nelson, Gerler, \& Narens, 1984; Schacter, 1983; Schreiber \& D. L. Nelson, in press; Schwartz \& Metcalfe, 1992). A particularly vivid example of this phenomenon is the case of a person who has a tipof-the-tongue (TOT) experience. In this special case, a person is unable to recall the sought-after target information but nevertheless has a high feeling of knowing (FOK) for it (for a review, see Brown, 1991). On what kinds of factors are these feelings based, given that the targeted information is not accessible explicitly? Although the TOT example is a useful illustration, feelings of knowing are not limited to this special case. They represent a more general phenomenon, most likely occurring whenever people receive a memory prompt (Koriat, 1993; Reder \& Ritter, 1992). Essentially they are predictions of future memory performance, and they may play an important functional role in memory. Various researchers have proposed that such predictions are used to regulate the flow of processing during retrieval. For example, given a moderate to high FOK computed during the early stages of retrieval, a person may decide to initiate a more extensive search of memory. Given a very low FOK, a search may not be ini-

Some of the data reported in this article also were reported at the 35 th annual meeting of the Psychonomic Society, November 1994, St. Louis. Correspondence should be addressed to T. Schreiber, Department of Psychology, University of Kansas, 426 Fraser Hall, Lawrence, KS 66045 (e-mail: schreibe@kuhub.cc.ukans.edu).

-Accepted by previous editor, Geoffrey R. Loftus tiated at all (Reder, 1988; Reder \& Ritter, 1992). FOKs also may be used as a basis for deciding when search should be terminated (Koriat, 1993; T. O. Nelson et al., 1984; T. O. Nelson, Leonesio, Shimamura, Landwehr, \& Narens, 1982; T. O. Nelson \& Narens, 1990). According to this view, FOKs are updated periodically during search and provide an indication of whether search should continue.

The question of what specific functional roles FOKs may play is still open, but, in the present context, an even more fundamental question is, What factors influence them? This article reviews some of the research that has addressed this question, with the focus initially being on studies showing that FOKs are particularly sensitive to information directly tied to the test cue. As will be explained in more detail, this research is consistent with the cue effectiveness hypothesis, which assumes that FOKs are based on evaluating the degree to which the test cue will be effective in producing the studied target item. More important, the article reports three new experiments that show that the cue effectiveness hypothesis provides an incomplete account of FOK effects. One of the key variables manipulated in each experiment was target set size, which refers to the number of preexisting connections a studied target word has to other words in long-term memory. Many studies have shown that target words connected to fewer related items are recalled with a higher probability than those connected to more related items, presumably because of the competition that is produced when memory for the target is searched (see, e.g., D. L. Nelson, 1989; D. L. Nelson, Bajo, \& Canas, 1987; D. L. Nelson, Bennett, Gee, Schreiber, \& McKinney, 1993; D. L. Nelson, Gee, \& Schreiber, 1992; D. L. Nelson \& Schreiber, 1992; D. L. Nelson, Schreiber, \& Holley, 1992; D. L. Nelson, Schreiber, \& McEvoy, 1992). With fewer 
items there is less competition when attempting to search for the target. The critical question is whether FOKs are sensitive to this kind of competition? This question is important, because the presence of target set size effects in any task indicates that information directly tied to the representation of the target played a role. As such, examining the effect of target set size on FOKs provides an opportunity to evaluate the cue effectiveness hypothesis.

\section{Feelings of Knowing and Cue-Based Sources of Information}

One of the more intriguing findings in investigations of FOK judgments is that they are not always based on the same factors that influence memory performance. FOKs appear to be particularly sensitive to cue-based sources of information, but that is not always the case for other tasks, such as recall (see, e.g., Metcalfe, 1993; Metcalfe, Schwartz, \& Joaquim, 1993; Reder \& Ritter, 1992; Schwartz, 1994; Schwartz \& Metcalfe, 1992). Schwartz and Metcalfe compared the effects of the degree of cue encoding (i.e., cue familiarization) and of the degree of target encoding on FOK ratings and cued recall. To manipulate degree of cue encoding, subjects were familiarized with some of the test cues beforehand in separate tasks. They found that subsequent FOK ratings were more positive when people had encoded the cues in these other tasks than when they had not encoded the cues. At the same time, recall was uninfluenced by the degree of cue encoding. Apparently FOK ratings, unlike recall, are influenced by a person's ability to recognize the test cue as a result of prior experience with it. Schwartz and Metcalfe also found that FOK ratings were not influenced by the degree of target encoding, whereas recall was influenced by this factor. Together, these findings represent dissociations between FOKs and actual knowing, and such dissociations have been obtained several times (see, e.g., Metcalfe et al., 1993; Reder \& Ritter, 1992). Both tasks, knowing ratings and recall, apparently can be based on different sources of information, with FOKs being particularly sensitive to cue-based sources.

The assumption that cue-based sources of information are important for FOKs also is compatible with other findings. Rather than manipulating the degree of cue encoding, Schreiber \& D. L. Nelson (in press) manipulated cue set size, which refers to the number of preexisting connections a test cue has to different words in long-term memory. Using association norms, they measured preexisting cue set size for various types of cues, such as taxonomic category names, rhymes, and meaning-related associates. Cues linked to different-sized sets of related items then were given as extralist cues in recall and FOK tasks. One important finding was that both tasks were influenced by cue set size. People invariably made higher knowing ratings the smaller the set of items that was linked to the test cue. Cue set size also had a similar pattern of effects in recall. As with knowing ratings, recall also was more likely when fewer items were linked to the test cue. Apparently, both FOKs and recall are sensitive to the number of competing items activated by the test cue.
With fewer items, there is less competition with the target, and FOKs are higher and recall is more likely than is the case with a greater number of competing items.

Although cue set size has similar effects on the magnitude of FOK ratings and on recall, Schreiber and Sergent (1998) discovered some interesting differences between these tasks. Both judgment and recall latencies were measured, and the findings indicated a different time course associated with each task. In recall, response times (RTs) always were much faster for cues linked to smaller sets than for those linked to larger sets. This finding was consistent with prior work suggesting that people sample competing items that are related to the test cue and that, with fewer competing items, the probability of sampling the target is higher and the time needed to retrieve it is faster than with a greater number of competing items (D. L. Nelson, Gee, \& Schreiber, 1992; D. L. Nelson, McEvoy, \& Bajo, 1988; D. L. Nelson, Schreiber, \& McEvoy, 1992). A different pattern of RTs was found for FOK ratings. These ratings always were made in substantially less time than was needed for recall, but there was never any timing advantage for cues linked to smaller sets. FOK ratings consistently were made in about the same amount of time, regardless of cue set size. These timing findings suggested that different processes may have been involved in each task; an issue that becomes important later on in this paper.

For now, the most important point is that both the findings obtained by Schwartz and Metcalfe (1992) and others involving manipulations of cue familiarization and the findings obtained by Schreiber and D. L. Nelson (in press) and Schreiber and Sergent (1988) involving manipulations of cue set size underscore the importance of cue-based sources of information in the FOK task. They also suggest that FOKs are not always based on the same kinds of processes as are other memory tasks, such as cued recall. One way to summarize these two sets of findings is to assume that FOKs provide a rapid means for evaluating cue effectiveness and that both cue familiarization and competition play independent roles in this evaluation. The first component of the cue effectiveness hypothesis assumes that, when prior acts of encoding cause the test cue to be perceived as more familiar and to be recognized, people are more likely to attribute to it effectiveness in producing the target later on (see, e.g., Metcalfe, 1993; Metcalfe et al., 1993; Reder \& Ritter, 1992; Schwartz, 1994; Schwartz \& Metcalfe, 1992). As such, the more strongly the cue was encoded in the past as a result of rehearsal processes or other acts of encoding, the greater its perceived familiarity and the more positive the FOK. As shown by Schwartz and Metcalfe and by others, cue familiarity can produce illusions of cue effectiveness, because increments in cue familiarity do not always lead to increments in memory performance. Independent of familiarity, evaluations of cue effectiveness also are determined partly by the amount of competition produced when the test cue activates directly connected items (Schreiber \& D. L. Nelson, in press; Schreiber \& Sergent, 1998). When fewer competing items are activated, there is less competition, 
and the cue is judged as being more effective than it is when more competing items are activated. As a consequence, FOKs are higher when the test cue is connected to a smaller set of competing items in long-term memory than they are when it is connected to a larger set of items.

\section{Feelings of Knowing and Target Set Size}

Does the cue effectiveness hypothesis, as defined in the present context, provide an adequate account of FOK effects? Specifically, are FOKs only influenced by information tied directly to the test cue, or can they also be influenced by information that is tied directly to the representation of the studied target word? Although at least some prior research is consistent with the cue effectiveness hypothesis, one problem is that the types of manipulations directed toward the target have been limited. A number of studies have manipulated the degree of target encoding in order to determine whether target-based sources influence FOKs and have failed to find any consistent effects (see, e.g., Metcalfe et al., 1993; Reder \& Ritter, 1992; Schwartz \& Metcalfe, 1992). However, it is possible that FOKs may be more sensitive to certain types of information about the target and less sensitive to others. For example, FOKs may be less sensitive to how strongly the target was encoded, but they may be more sensitive to other factors, such as the amount of competition produced when words that are directly linked to the target are activated.

To evaluate this possibility, the present experiments varied the number of associates linked to the studied target word. Some experiments also varied the degree of target encoding, but the main manipulation was target set size. By including manipulations of target set size, the focus was on associates of the target word rather than on associates of the test cue, as in Schreiber and D. L. Nelson (in press; Schreiber \& Sergent, 1998). The effect of target set size was examined in the context of a relatively simple memory paradigm in which the subjects were given test cues that were not presented during the study phase. In this associative cuing paradigm, the subjects first are shown familiar words during study, and they then receive related words as extralist cues in the recall and FOK phases. This paradigm is different from the usual memory paradigm for investigating FOK effects, because the test cue was not presented during the study trial. One important reason for using this procedure was to isolate factors linked to the study and test trial, and a second reason was that it is ideally suited to explore the role of preexisting memories in an episodic memory task. The main manipulation in each experiment consisted of target set size, with one half of the studied items being linked to a smaller number of related words and the remaining one half being linked to a larger number of related words. The specific purpose was to determine whether target set size would have effects on FOK ratings and recall similar to those of cue set size. If FOKs are sensitive to competition between the target and other items linked to it, higher FOK ratings should be obtained for targets linked to a smaller number of related items than for those linked to a larger number of related items. Such a finding would have important implications for the cue effectiveness hypothesis, because it would indicate that FOKs are not determined exclusively by cuebased sources of information. On the other hand, if FOKs are based exclusively on cue-based sources of information, target set size should not have any effect.

Would the effects of target set size follow a similar pattern in the FOK task and in recall? Many studies have shown that targets linked to a smaller number of competing items are recalled with a higher probability than those linked to a greater number of competing items (see, e.g., D. L. Nelson, 1989; D. L. Nelson, Bajo, \& Canas, 1987; D. L. Nelson, Bennett, Gee, Schreiber, \& McKinney, 1993; D. L. Nelson, Gee, \& Schreiber, 1992; D. L. Nelson \& Schreiber, 1992; D. L. Nelson, Schreiber, \& Holley, 1992; D. L. Nelson, Schreiber, \& McEvoy, 1992; for reviews, see D. L. Nelson, 1989; D. L. Nelson, Schreiber, \& McEvoy, 1992). In addition, timing findings indicate that targets linked to smaller sets are recalled more quickly than those linked to larger sets (D. L. Nelson, Gee, \& Schreiber, 1992). Essentially, these target set size findings in recall parallel cue set size findings (McEvoy \& Holley, 1990; D. L. Nelson \& Bajo, 1985; D. L. Nelson, Canas, Bajo, \& Keelean, 1987; D. L. Nelson \& Friedrich, 1980; D. L. Nelson \& McEvoy, 1979; D. L. Nelson et al., 1988; D. L. Nelson, McEvoy, \& Friedrich, 1982; D. L. Nelson \& Schreiber, 1992; D. L. Nelson, Schreiber, \& McEvoy, 1992), and one way to explain both effects is by assuming that the activation of related items produces competition. For example, the PIER ${ }^{1}$ model was designed, in part, to explain cue and target set size effects in recall, and it emphasizes the role of competition (D. L. Nelson, Schreiber, \& McEvoy, 1992). To explain target set size effects, PIER makes separate assumptions about processing that takes place during the study phase and processing that takes place during the testing phase. Presumably, when a target word is studied, preexisting connections to closely related words are activated or primed very rapidly and in parallel. The purpose of this activation process is to increase the accessibility of information that may become relevant. When a related word is presented as a cue during the testing phase, connections to the target and to neighboring items become activated. Once the target is activated by the test cue, it becomes the source of further activation, spreading to items linked directly to its representation (cf. Anderson, 1983; Collins \& Loftus, 1975; Kintsch, 1988). As such, the target can reactivate connections to closely related items during testing that originally were activated during the study trial (D. L. Nelson, Schreiber, \& McEvoy, 1992).

Although the cue presented during the testing phase is the initial source of activation, a pool of candidate responses is created by the spreading of activation along links to items connected to it and then further to more distant items. As such, associates of the test cue as well as associates of the target become involved as activation spreads. The pool of words comprising the activated set then can be searched, and, in PIER, search consists of a 
serial sampling process. Sampling continues until the target has been identified or until the stop search criterion is reached. Search should occur if the task requires the selection of one particular representation from the pool of activated representations, as in recall. Under these conditions, the items in the pool compete with the target for selection. When there are fewer activated representations in the pool, there is less competition for selection between the target and other representations; the probability of selecting the target is higher, and the time to select the target is faster than they are when there are a greater amount of activated representations in the pool (cf. Gillund \& Shiffrin, 1984; Raaijmakers \& Shiffrin, 1981). Put simply, the parallel findings that are obtained when cue set size and target set size are varied are consistent with the hypothesis that both effects are caused by accessing competing items (D. L. Nelson, Schreiber, \& McEvoy, 1992). If FOKs, like recall, also are sensitive to the amount of competition produced by items linked to the representation of the target, FOKs should be higher when fewer items are linked to the target than they are when a greater number of items are linked to the target. On the other hand, if FOKs are sensitive only to competition between concepts tied directly to the test cue rather than to the target, target set size should not have any effect.

\section{The Experiments}

Each experiment involved manipulations of target set size, and each used the associative cuing paradigm. In addition to manipulating target set size, Experiment 1 compared two different types of FOK tasks. In the standard FOK task, the subjects were given a cued recall test immediately after the study phase. The cues that failed to elicit the correct target word then were reshown, and the subjects made FOK ratings. The sequence of events in the prediction-of-knowing (POK) condition was different from that in the FOK condition. The subjects made ratings immediately after study on all items rather than only on unrecalled items, and then they performed the cued recall task. Also, unlike in the FOK task, the subjects were encouraged to make their ratings as rapidly as possible, relying on their initial impressions. The purpose of exerting time pressure was to determine whether the effects of target set size would differ as a function of instructions emphasizing fast responses. The purpose of Experiment 2 was to determine whether knowing ratings would be influenced by different encoding tasks and whether comparable target set size effects would be found for each of these tasks. During study, the subjects were asked to classify words with respect to concreteness or to name their vowels (i.e., level of processing), and, after performing these tasks, they were tested with meaningfully related words as cues. Experiment 3 also varied both target set size and encoding task, but it used ending sounds rather than meaningfully related words as cues. The purpose was to determine whether meaning-related target set size effects would be obtained if the attention of subjects was oriented toward word sound rather than to word meaning during the testing phase.

\section{EXPERIMENT 1}

The main purpose of this experiment was to determine whether the number of associates linked to studied target words has any effect on FOK and POK ratings. As with the cue set size experiments conducted by Schreiber and Sergent (1998), RT measurements were obtained in this experiment to supplement FOK ratings and recall accuracy measures. The primary reason for obtaining these measurements was to determine whether the time course for target set size resembles the time course for cue set size (D. L. Nelson et al., 1988; Schreiber \& Sergent, 1998). Previous findings involving manipulations of target set size parallel findings involving manipulations of cue set size and indicate that recall is more likely and RTs in recall are faster for targets linked to smaller associative sets than for those linked to larger sets (see, e.g., D. L. Nelson, Gee, \& Schreiber, 1992), and it was expected that the same pattern would be obtained in the present experiment. This pattern of RTs is consistent with the assumption that target set size effects in recall, like cue set size effects, arise as a result of sampling processes used during search rather than as a result of parallel activation processes. The critical question was whether a similar time course would be obtained for FOK and POK ratings.

Mainly on the the basis of findings involving manipulations of cue set size, it was expected that, if target set size had any effect on the magnitude of knowing ratings, there should not be any effect on the time needed to make the ratings. This pattern would be consistent with the view that knowing ratings are sensitive to competition that arises when related items are activated in parallel. ${ }^{2}$ It would be inconsistent with the view that knowing ratings involve the same kind of sampling processes as those used in recall. Although it was not expected that target set size would affect rating latencies, such effects should be obtained for recall. Because sampling processes are used in recall, less time should be needed to make a response when targets are linked to fewer items.

\section{Method}

Design and Subjects. The experimental design formed a $2 \times 2$ mixed model factorial. Target set size (small, large) was manipulated within subjects, and type of knowing rating task (FOK, POK) was manipulated between subjects. Sixty subjects participated in the experiment, with 30 randomly assigned to each rating task. All of the subjects were selected from courses in introductory psychology at the University of South Florida, Tampa.

Materials. Most of the test cues and targets were based on two lists used in a previous recall study (D. L. Nelson, Schreiber, \& Holley, 1992, Experiment 4). A normative database consisting of approximately 3,500 words was used to construct the original list (D. L. Nelson, McEvoy, \& Schreiber, 1995). In the norming task, many groups of subjects $(n=100-200)$ were given a large number of different stimulus words, with instructions to write the first word to come to mind that was meaningfully related to or strongly associated with each stimulus word. The norms were used to estimate both the strength of the preexisting cue-to-target relationship and associative set size (see D. L. Nelson \& Schreiber, 1992, and D. L. Nelson, Schreiber, \& Holley, 1992, for additional details and rationale). In the present experiment, most of the cues and targets from Lists 1 and 2 of D. L. Neison, Schreiber, and Holley were combined, re- 
sulting in a total of 44 cues and targets. Some of the items from the recall study were not included in this experiment, because some cues from one list were related to targets from the other list. The lists were combined as much as possible, rather than used separately, to ensure relatively large samples of recalled and unrecalled items for each subject. Half of the targets had relatively small associative sets, averaging 6.68 associates $(S D=1.46)$, and the remaining half had larger associative sets, averaging 19.01 associates $(S D=2.25)$. Other attributes of the test cue and target were equated for each level of target set size in order to ensure that such attributes would not be confounded with target set size. Of these attributes, only cue set size has been shown to influence FOKs (Schreiber \& D. L. Nelson, in press). Other factors were equated either because they could conceivably affect cue recognition (e.g., cue frequency, cue concreteness) or because they are known to affect recall (e.g., cue-totarget strength, target concreteness, target frequency). For each level of target set size, the probability of producing the target in the absence of study (i.e., cue-to-target strength) averaged $.22(S D=$ $.13)$ and $.21(S D=.14)$, cue set size averaged $12.27(S D=3.77)$ and $13.45(S D=3.95)$, cue concreteness averaged $5.10(S D=1.09)$ and $5.37(S D=1.27)$, printed frequency of the cues averaged $112.54(S D=275.33)$ and $92.09(S D=272.03)$ occurrences per million (Kučera \& Francis, 1967), target frequency averaged 48.64 $(S D=62.28)$ and $64.04(S D=85.87)$ occurrences per million, and target concreteness averaged $5.20(S D=1.05)$ and $5.26(S D=$ 1.20 ). Statistical analyses indicated that none of these pairs of means were significantly different, with most $F_{\mathrm{s}}<1$.

Procedure. The procedure for the FOK and POK conditions was similar to that in prior work involving the associative cuing paradigm (see, e.g., Schreiber \& D. L. Nelson, in press). The FOK condition included study, cued recall, and FOK ratings. During the study phase, the target words were presented on a computer screen at a rate of $4 \mathrm{sec}$ per word. The subjects were given intentional learning instructions, but the specific nature of subsequent phases was not mentioned. In the FOK condition, a cued recall test was given immediately after study. The cue words were displayed on the computer screen one at a time, and the subjects were asked to recall the related study word. Whenever the subjects could not recall the study word and did not want to guess, they were asked to say the word "Next." When giving their recall response verbally, they were asked to press the space bar at the same time so that RTs could be measured. After recall, the cues that did not elicit a correct response were reshown, and the subjects made knowing ratings, using a sixpoint scale. The subjects were informed that only cues for unrecalled items would be reshown. In the rating scale that people were asked to use, a rating of six represented the most positive FOK, and one represented the least positive. Once again, the subjects gave their verbal responses and pressed the spacebar simultaneously.

The procedure in the POK condition was similar to the procedure in the FOK condition, except that the order of the recall and rating phases was reversed. Thus, in the POK condition, the subjects studied targets, made FOK ratings on all items, and then performed the cued recall test. The instructions for the rating phase in the POK condition were adapted from Reder $(1987,1988)$. The subjects were encouraged to make their ratings as quickly as possible; they were asked to rely on their initial impressions or sense of whether they knew the related study word. FOK ratings, on the other hand, were self-paced. All items were presented in a different random order for every phase and for every subject.

\section{Results and Discussion}

The rating data will be discussed first, followed by the recall and RT data. The criterion for significance was set at $p<.05$ in all experiments. Two separate analyses of variance (ANOVAs) were conducted to facilitate comparison with prior studies. The first analysis focused on unrecalled items in the POK and FOK conditions, and the second analysis focused on both recalled and unrecalled items in the POK condition alone. The factors in the first analysis were knowing rating task and target set size. The factors in the second analysis were target set size and type of recall response (recalled, unrecalled).

Knowing ratings. The left and middle portions of Figure 1 show mean knowing ratings for unrecalled items as a function of rating task and target set size. The figure shows that ratings involving unrecalled items were higher when targets were linked to smaller associative sets than when they were linked to larger associative sets, and the means for these conditions were, respectively, 3.54 and 3.11. The difference between targets with smaller and larger sets was apparent in both knowing rating conditions, but this difference was somewhat larger in the POK condition (3.48 vs. 2.89 ), as compared with the FOK condition ( 3.60 vs. 3.33 ). In addition, mean ratings tended to be higher overall in the FOK condition $(3.46)$ than in the POK condition (3.18). The statistical analysis indicated that the effect of target set size was significant $[F(1,58)=$ $\left.28.40, M S_{\mathrm{e}}=0.19\right]$, but that neither the effect of type of knowing rating $\left[F(1,58)=2.82, M S_{\mathrm{e}}=1.43\right]$ nor the interaction between task and target set size were reliable $[F(1,58)=3.73]$. These results indicate that both POK and FOK ratings for unrecalled items are influenced by the number of associates linked directly to the target, and this finding is inconsistent with the hypothesis that knowing ratings are sensitive only to cue-based sources of information.

The results of the separate analysis involving only the POK task and the ratings on unrecalled as well as recalled items also indicated that the effect of target set size was significant $\left[F(1,29)=21.13, M S_{\mathrm{e}}=0.25\right]$,

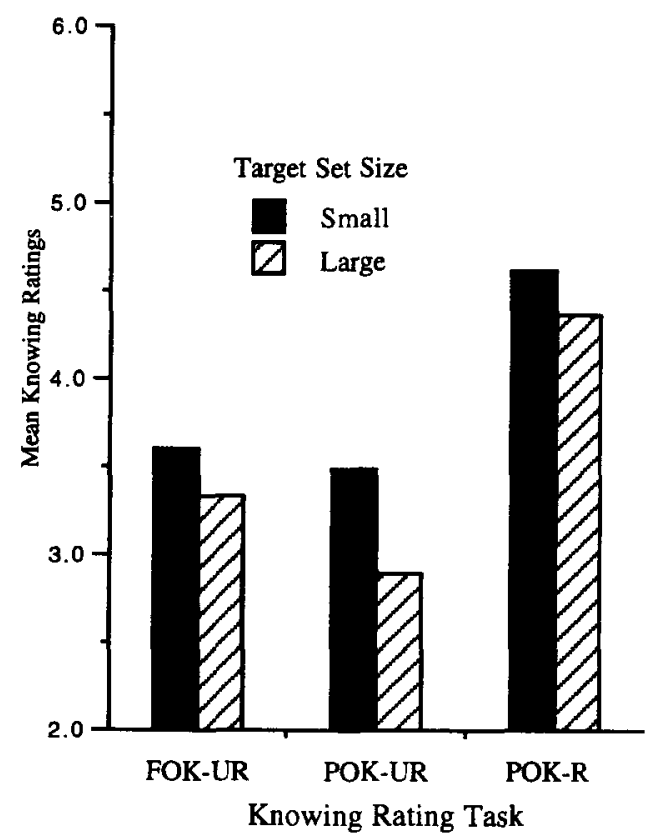

Figure 1. Mean feeling-of-knowing (FOK) and prediction-ofknowing (POK) ratings for unrecalled (UR) and recalled items $(R)$ in Experiment 1. 
with higher ratings for targets linked to smaller sets, as compared with those linked to larger sets (see the middle and rightmost portions of Figure 1). The means for targets with smaller and larger sets were, respectively, 4.04 and 3.62. Mean ratings also were higher for recalled items than for unrecalled items $\left[F(1,29)=87.05, M S_{\mathrm{e}}=\right.$ 0.58 ], and the means for these items were 4.48 and 3.18 . The interaction between type of recall response and target set size also was reliable $\left[F(1,29)=5.43, M S_{\mathrm{e}}=\right.$ 0.83 ]. The difference between mean ratings for targets linked to smaller compared with larger sets of associates was significant both for recalled and for unrecalled items, and this difference was somewhat larger for unrecalled items (least significant difference [LSD] $=.33$ ).

Probability of recall. Mean probabilities of recall are displayed in the first two rows of Table 1 . Replicating findings from previous research (e.g., D. L. Nelson, Schreiber, \& McEvoy, 1992, and many others), the recall findings indicated that targets linked to smaller associative sets were more likely to be recalled than targets linked to larger associative sets $\left[F(1,58)=95.02, M S_{\mathrm{e}}=0.01\right]$. The means for these conditions were .53 and .36 . In addition, the probability of recall was slightly higher in the FOK $(.49)$ than in the POK $(.41)$ condition $[F(1,58)=$ 5.83, $\left.M S_{\mathrm{e}}=0.04\right]$. The difference between recall in the FOK and POK conditions most likely was due to the delay between study and recall being greater in the POK than in the FOK conditions. The interaction between type of rating task and target set size was not reliable, with $F<1$.

Response times. As with the rating data, two analyses were conducted. The first focused on unrecalled items, and the factors were knowing rating task, target set size, and test type (rating, recall). The factors in the second analysis, focusing both on recalled and on unrecalled items in the POK condition alone, were target set size, type of recall response (recalled, unrecalled), and type of test. Also, outliers that were more than 2.0 standard deviations from the subjects' mean were replaced with the next highest score that was within this range (Tabachnick \& Fidell, 1983, p. 76). Although the most important effect concerned the interaction between target set size and type of test, other findings involving main effects will be presented first.

Mean RTs for unrecalled items in the FOK and POK conditions are shown in the left and middle panels of Figure 2. Overall, the pattern of findings resembled that

Table 1

Mean Probabilities of Correct Recall for Experiments 1-3

\begin{tabular}{|c|c|c|c|}
\hline \multirow[b]{2}{*}{ Experiment } & \multirow[b]{2}{*}{ Task } & \multicolumn{2}{|c|}{ Target Set Size } \\
\hline & & Small & Large \\
\hline \multirow[t]{3}{*}{1} & Knowing rating & & \\
\hline & Feeling of knowing & .58 & .41 \\
\hline & Prediction of knowing & .49 & .32 \\
\hline \multirow[t]{3}{*}{2} & Encoding & & \\
\hline & Concreteness classification & .66 & .48 \\
\hline & Vowel naming & .36 & .25 \\
\hline \multirow[t]{3}{*}{3} & Encoding & & \\
\hline & Concreteness classification & .63 & .54 \\
\hline & Vowel naming & .49 & .37 \\
\hline
\end{tabular}

obtained in conjunction with manipulations of cue set size (Schreiber \& Sergent, 1998). RTs were faster for knowing ratings (2.93), as compared with recall (6.63), when the targets were linked to smaller associative sets (4.55) than they were when the targets were linked to larger associative sets (5.01), and they were faster for the POK condition (5.96) than for the FOK condition (3.60). The statistical analysis indicated that the main effects of type of test $\left[F(1,58)=117.75, M S_{\mathrm{e}}=6.98\right]$, target set size $\left[F(1,58)=12.84, M S_{\mathrm{e}}=0.01\right]$, and knowing rating condition $\left[F(1,58)=27.45, M S_{\mathrm{e}}=12.21\right]$ were reliable. The statistical analysis also indicated that two interactions were reliable. First, as in the findings of the cue set size experiments, there was a magnitude interaction between type of test and knowing rating condition $[F(1,60)=$ 15.27]. Both FOK and POK ratings were made in substantially less time than was required to provide a response on the recall test. The source of the interaction was that differences between rating and recall latencies were larger in the FOK condition (3.44 vs. 8.48) than in the POK condition (2.41 vs. 4.78$)$. The finding that knowing ratings were made more quickly overall than was providing a response in recall replicates previous findings (see, e.g., Reder, 1988; Schreiber \& Sergent, 1998, Experiments 1-3).

The second and most important interaction was between target set size and type of test $[F(1,58)=21.12$, $\left.M S_{\mathrm{e}}=0.93\right]$. Pooled across type of knowing rating task, RTs on unrecalled items averaged $2.98 \mathrm{sec}$ for targets with smaller associative sets and $2.87 \mathrm{sec}$ for targets with larger sets. In recall, the means were 6.11 and $7.14 \mathrm{sec}$ for targets with smaller as compared with larger associative sets. Thus, for knowing ratings, responses were made in about $0.12 \mathrm{sec}$ more time for targets with smaller as compared to larger sets, whereas, in recall, RTs were over $1.00 \mathrm{sec}$ faster for targets with smaller sets (LSD $=.35$ ). Essentially, the standard effect, involving a direct relation between target set size and RTs, was obtained for recall, but there was little difference for targets with smaller and larger sets in the knowing rating tasks. This interactive pattern is similar to the pattern found for the cue set size experiments; it is more consistent with the hypothesis that FOKs are sensitive to the competition between a target and neighboring concepts that arises as a result of parallel activation processes than with the hypothesis that they are sensitive to competition that arises as a result of the kinds of sampling processes that presumably are utilized in recall.

A similar pattern of findings was obtained in the second analysis, involving both unrecalled and recalled items in the POK condition alone. Mean RTs for these conditions are shown in the middle and right panels of Figure 2. Once again, the interaction between target set size and type of test was significant $[F(1,29)=31.07$, $\left.M S_{\mathrm{e}}=0.33\right]$. For POK ratings, RTs averaged $2.50 \mathrm{sec}$ for targets with smaller associative sets and $2.26 \mathrm{sec}$ for targets with larger sets. In recall, the means were 3.69 and $4.28 \mathrm{sec}$ for targets with smaller as compared with larger sets. The three-way interaction between target set size, 

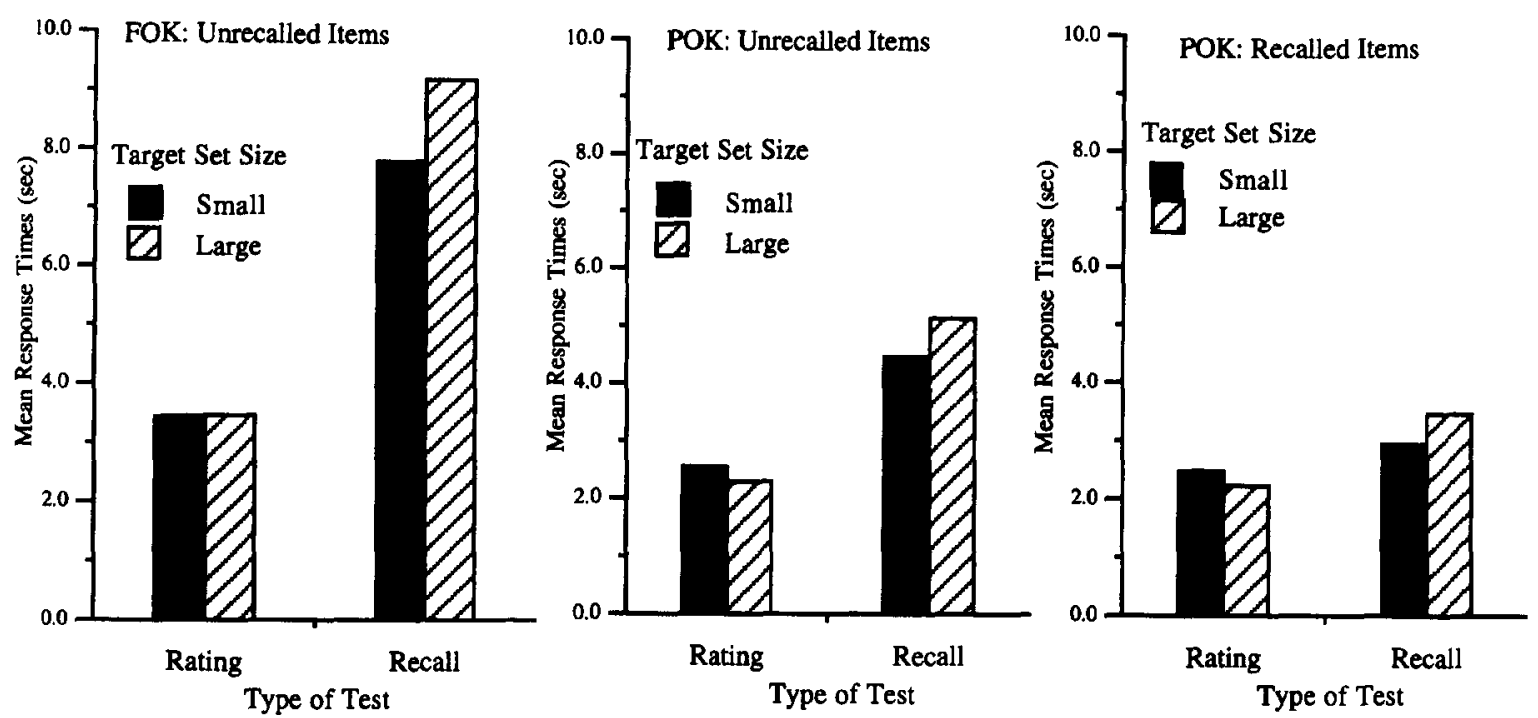

Figure 2. Mean response times for feeling-of-knowing (FOK) and prediction-of-knowing (POK) ratings and for recall (Experiment 1).

type of test, and correctness of the response made in recall was not reliable, with $F<1$.

The most important findings concerned the relation between target set size and the magnitude of knowing ratings and the relation between target set size and RTs when making these ratings. One finding was that the magnitudes of both FOK and POK ratings were significantly higher when studied words were linked to smaller as compared with larger associative sets. This finding is compatible with the hypothesis that knowing ratings are sensitive to the amount of competition between a studied word and the neighboring associates linked directly to that word as a result of preexisting connections. The finding is incompatible with any hypothesis that assumes that FOKs are determined exclusively by cue-based sources of information, because target set size involves information directly linked to the studied word. A second finding was that rating latencies did not differ appreciably for studied words linked to different sized sets but that recall latencies were substantially faster when targets were linked to smaller sets. Both findings indicate that knowing ratings and recall follow somewhat different time courses. Knowing ratings may be more sensitive to competition arising as a result of activation processes than to competition arising as a result of sampling processes.

\section{EXPERIMENT 2}

The purpose of this experiment was to determine whether knowing ratings are influenced by different encoding tasks and whether comparable target set size effects would be found for each of these tasks. During study, the subjects performed incidental learning tasks that oriented them to different aspects of the target words. In one task, they were asked to think about the meaning of the target words and to classify them with respect to concreteness. Other subjects were asked to name the vowels in the target words. After performing these tasks, they were tested with meaningfully related words as cues, as before. Type of encoding task (concreteness classification, vowel naming) was crossed with target set size (small, large). The most important reason for including different encoding tasks was to determine whether target set size effects comparable to those found in Experiment 1 also would be obtained using the concreteness classification and vowel-naming tasks. Many recall experiments have shown that target set size effects do not depend on the use of particular encoding strategies. For example, in recall, equivalent target set size effects are found, regardless of whether target words were encoded under intentional learning instructions or whether subjects rated them for pleasantness or concreteness, named their vowels, or read target words in the context of sentences (D. L. Nelson, Bajo, \& Casanueva, 1985; D. L. Nelson, Gee, \& Schreiber, 1992; D. L. Nelson, Schreiber, \& Holley, 1992; D. L. Nelson, Schreiber, \& McEvoy, 1992). Apparently, attention to meaning during study is not required in order for associatively related words to become activated during encoding and to influence performance in recall. The question is whether this would also be the case for knowing ratings. To evaluate this possibility, type of encoding task was crossed with target set size.

A second reason for including different encoding tasks was to determine whether knowing ratings overall are influenced by the degree to which targets are encoded during study. Prior studies have failed to find that knowing ratings are sensitive to degree of target encoding (see, e.g., Reder \& Ritter, 1992; Schwartz \& Metcalfe, 1992), and the purpose was to determine whether this null effect would occur with what should be a relatively effective encoding manipulation. Concreteness classifications involve greater attention to meaning than does naming the vowels of words and should produce sizable effects in recall. These tasks have been used many times in other re- 
call experiments and consistently produce fairly large differences in performance (see, e.g., Craik \& Tulving, 1975 ; D. L. Nelson, Schreiber, \& Holley, 1992; D. L. Nelson, Schreiber, \& McEvoy, 1992).

With the exception of differences relating to the different encoding conditions, the procedure employed in the present experiment was similar to the procedure employed in Experiment 1. Once again, extralist cues were used, and the characteristics of these cues, such as cue set size and cue-to-target strength, were equated for each level of target set size. Aside from manipulating encoding instructions, the main procedural difference between Experiment 1 and this experiment was that only the POK task was used. There were several reasons for only including the POK task. First, the results of Experiment 1 showed that both POK and FOK ratings were affected in the same way by target set size. Second, in the experiments involving manipulations of cue set size reported by Schreiber and D. L. Nelson (in press) and Schreiber and Sergent (1998), both rating tasks always produced a similar pattern of findings. On the basis of the similar findings obtained with both rating tasks in these studies, new information probably would not be obtained by including both tasks once again. Third, in the POK task, knowing ratings are made on all items and are not contingent on items being unrecalled, as is the case with the standard FOK task. Fourth, the fact that POK ratings are made on all items allows an examination of ratings on recalled items, unrecalled items, or both kinds of items pooled. Thus, the use of the POK task not only provides information that is available with the standard task, in terms of analyses based on unrecalled items, but also provides information that cannot be obtained with the standard FOK rating task, in terms of analyses based on recalled items. Finally, because the POK task emphasizes response speed, it provides a stronger test of the cue effectiveness hypothesis. Reder and Ritter (1992) speculated that, when subjects are encouraged to make very rapid knowing ratings, access to target-related information should be particularly unlikely. For all of these reasons, only POK ratings were used in this experiment and in Experiment 3.

\section{Method}

Design and Subjects. The experimental design consisted of a $2 \times 2$ mixed model factorial with target set size (small, large) manipulated within subjects and type of encoding task (concreteness classification, vowel naming) manipulated between subjects. Fiftytwo subjects, from the same sources as before, participated in the experiment, with an equal number randomly assigned to each encoding condition.

Materials. A new set of materials was constructed from the same normative database as that described in Experiment 1, and these materials are shown in Appendix A. Two separate lists of 44 cues and targets were constructed, and an equal number of subjects was randomly assigned to each list. Half of the targets in each list had smaller associative sets, and the remaining half had larger associative sets. Targets with smaller sets averaged 6.75 associates $(S D=$ 1.50), and targets with larger associative sets averaged 19.68 associates $(S D=3.10)$. As before, other attributes of the test cue and target were equated in each condition of target set size. For each level of target set size, cue set size averaged $12.14(S D=3.52)$ and $13.25(S D=4.04)$, preexisting cue-to-target strength averaged 0.21 $(S D=0.09)$ and $0.20(S D=0.09)$, cue concreteness averaged 4.68 $(S D=1.35)$ and $4.38(S D=1.74)$, printed frequency of the cues averaged $56.59(S D=239.14)$ and $49.18(S D=193.56)$, target concreteness averaged $4.96(S D=1.50)$ and $4.52(S D=1.89)$, and the printed frequency of targets averaged $35.61(S D=53.37)$ and $56.41(S D=70.49)$. Statistical analyses involving these characteristics indicated that none of the differences between these means was reliable, with most $F \mathrm{~s}<1$.

Procedure. The procedure was identical to the procedure in Experiment 1 for the POK conditicn, with the exception of differences relating to the study instructions. In the present experiment, the subjects in the concreteness classification condition were asked to read each study word aloud and to classify each word as concrete, abstract, or neutral, depending on its concreteness. Usually, subjects in the concreteness condition are asked to make concreteness ratings instead of concreteness classifications. The present experiment involved concreteness classifications instead of ratings, because making ratings during study as well as in the POK phase potentially could cause interference. In the vowel-naming condition, the subjects were asked to read each study word aloud and to name its vowels. For both conditions, the instructions were incidental, in the sense that they did not mention any of the subsequent phases of the experiment nor the fact that the purpose of the experiment was to test memory and metamemory. All remaining aspects of the procedure were identical to the procedure used for the POK conditions in Experiment 1 .

\section{Results and Discussion}

Prediction-of-knowing ratings. Because this experiment did not include FOK ratings, only one analysis of the rating data was conducted. The variables in this analysis were target set size, encoding condition, and the correctness of the response made on the subsequent recall test. Figure 3 shows the means for these conditions. As indicated in the figure, POK ratings were higher for targets linked to smaller associative sets (3.86) than for targets linked to larger associative sets (3.48), and the statistical analysis indicated that the difference between these means was significant $\left[F(1,50)=25.51, M S_{\mathrm{e}}=0.28\right]$. This finding indicates that, as with Experiment 1 , the target set size effect was obtained. Other findings indicated that mean POK ratings were higher for targets that were recalled (4.37) than for those that were not recalled (2.97) and for targets encoded in the concreteness classification condition (4.02) than for those encoded in the vowelnaming condition (3.32). Both the effect of correctness of the response in recall $\left[F(1,50)=142.41, M S_{\mathrm{e}}=0.72\right]$ and the effect of encoding condition $[F(1,50)=12.90$, $\left.M S_{\mathrm{e}}=1.97\right]$ were significant sources of variance.

Target set size did not interact with either correctness of the recall response or encoding condition, with both $F$ s near unity. The lack of an interaction between these factors indicates that target set size effects in the POK task did not depend on the use of an encoding strategy that emphasized attention to the meaning of studied words, as compared with encoding strategies that emphasized the analysis of more perceptual characteristics. Presumably, as in recall, target set size effects for POK ratings are the result of relatively automatic processes. The sta- 

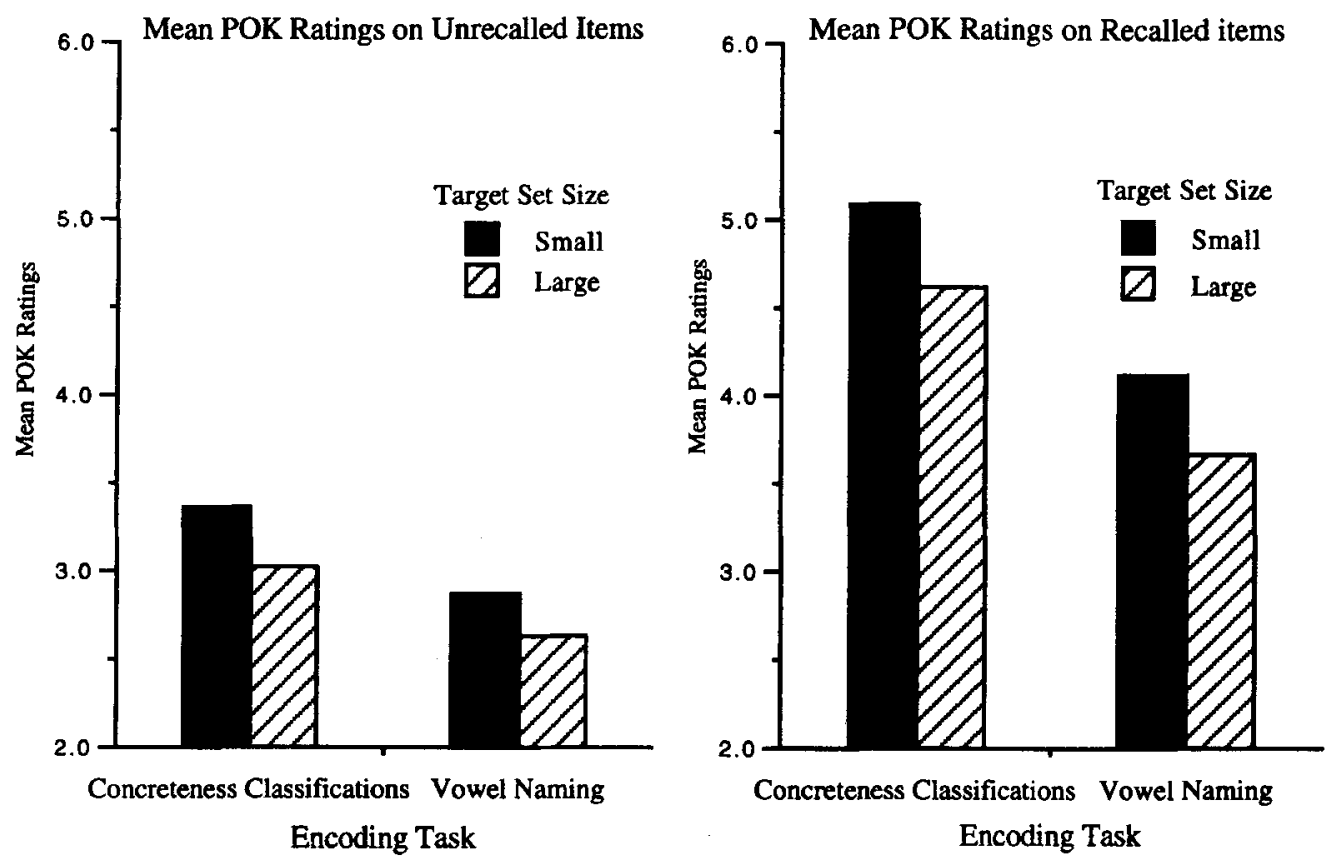

Figure 3. Mean prediction-of-knowing (POK) ratings (Experiment 2).

tistical analysis also indicated that the interaction between encoding condition and correctness was significant $[F(1,50)=4.97]$. As shown in Figure 3, for unrecalled items, mean ratings were higher in the concreteness condition (3.19) than in the vowel-naming condition (2.75). For recalled items, POK ratings also were higher in the concreteness condition (4.85) than in the vowel-naming condition (3.96), but the difference between these means was greater than the difference between comparable means involving unrecalled items. A two-tailed LSD of .47 indicated that the effect of encoding condition was significant for recalled items $(p<.0001)$, but this effect was not quite significant for unrecalled items $(p<.06)$. This interaction is consistent with the assumption that differences between different degrees of encoding might be more easily detected for items that subjects eventually can recall, as compared with those they cannot recall. The fact that an effect of encoding condition was obtained at all indicates that this manipulation may have been particularly effective.

Probabilities of recall. The third and fourth rows of Table 1 show the mean probabilities of recall. The probability of recall was higher for targets linked to smaller associative sets (.51), as compared with those linked to larger sets $\left[.37 ; F(1,50)=55.10, M S_{\mathrm{e}}=0.01\right]$. The mean also was higher in the concreteness classification condition (.57) than in the vowel-naming condition (.30), and the difference between these means also was significant $\left[F(1,50)=70.78, M S_{\mathrm{e}}=0.03\right]$. The interaction between these sources was not significant $[F(1,50)=2.79]$. Each of these effects replicates a very large number of prior studies. The relatively large difference between the mean for the concreteness classification condition and that for the vowel-naming condition also indicates that the encoding manipulation was fairly strong. Recall was nearly .30 higher for the concreteness condition than for the vowelnaming condition. Despite this relatively large difference, equivalent target set size effects were found for each encoding condition.

Response times. RTs were analyzed primarily to determine whether the interaction between target set size and type of test that was found in Experiment 1 could be replicated with the procedure and materials of the present experiment. The factors in the analysis were target set size, type of test, correctness of the response made in recall, and encoding condition. The effects were similar to those obtained in Experiment 1 and paralleled the prior findings involving manipulations of cue set size. Overall, mean RTs were faster for targets linked to smaller associative sets (2.79) as compared with those linked to larger associative sets (2.99), for POK ratings (2.43) as compared with recall (3.35), and for recalled items (2.32) as compared with unrecalled items (2.47). Mean RTs following concreteness classifications (2.87) were about the same as those following vowel naming (2.92). The statistical analysis indicated that the main effects of target set size $\left[F(1,50)=13.22, M S_{\mathrm{e}}=0.32\right]$, type of test $[F(1,50)=$ $\left.56.12, M S_{\mathrm{e}}=1.57\right]$, and correctness of the recall response $\left[F(1,50)=103.15, M S_{\mathrm{e}}=0.73\right]$ were reliable and that the effect of encoding condition was not $(F<1)$.

The most important finding involving RT measurements concerned the interaction between target set size and type of test. Experiment 1 showed that this interaction was significant, and, as indicated in Figure 4 , the same finding occurred here $\left[F(1,50)=32.56, M S_{\mathrm{e}}=\right.$ 0.28 ]. Mean RTs for POK ratings were comparable for 

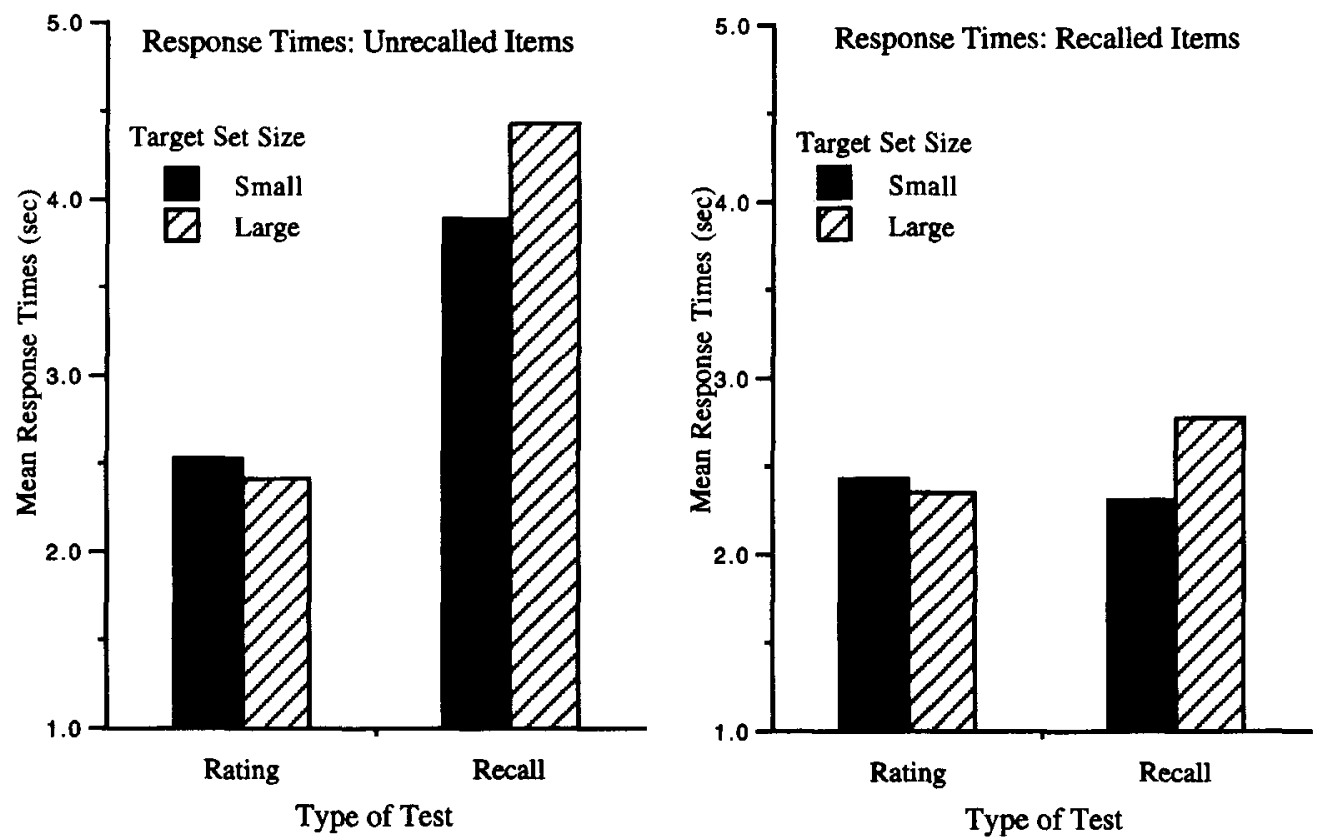

Figure 4. Mean response times for prediction-of-knowing (POK) ratings and for recall (Experiment 2).

targets linked to smaller associative sets (2.48) and for those linked to larger associative sets $(2.38)$. In the recall task, a different pattern emerged. In recall, RTs were faster for targets linked to smaller sets (3.10) than for those linked to larger sets (3.60). A two-tailed LSD of .30 indicated that the effect of target set size was not reliable for POK ratings but that the effect of target set size was significant for recall. No other higher level interactions involving target set size and type of test were reliable, including the three-way interaction with correctness of the recall response $(F<1)$ and the three-way interaction with encoding condition $[F(1,50)=1.95]$.

The findings showed that the magnitude of POK ratings was higher for studied words linked to smaller sets of associates, as compared with studied words linked to larger sets of associates, regardless of the kind of encoding strategy involved. Once again, these findings are not compatible with any hypothesis that assumes that FOKs are only sensitive to cue-based sources of information. In addition, equivalent target set size effects were found when the subjects classified words with respect to concreteness during study or named their vowels. The finding that target set size effects were obtained even when the subjects were not encouraged to attend to the meaning of studied words indicates that the activation of the associates of a target may have been the result of relatively automatic processes during encoding.

Other findings indicated that POK ratings were made about as quickly for targets with smaller associative sets as for those with larger associative sets. However, in recall, responses were made significantly more quickly for targets with smaller as compared with larger sets. Once again, this interactive pattern replicates the finding in Experiment 1 and parallels the findings in Schreiber and
Sergent (1998) involving manipulations of cue set size. Both results are consistent with the assumption that, when making POK ratings, people can sense the amount of competition arising as a result of activation processes. Because activation occurs in parallel, there were no time course differences in the rating tasks. In contrast, for recall, the faster RTs for targets linked to smaller numbers of competing associates than for those linked to larger numbers of associates is consistent with the assumption that sampling processes were utilized in this task. With fewer activated items, the sampling process is faster than it is with more activated items, because there are fewer competitors that can interfere with the sampling of the target.

\section{EXPERIMENT 3}

The results of Experiment 2 indicated that the number of associates linked to the target influences POK ratings, regardless of whether the encoding strategy during study emphasized attention to meaning or whether it emphasized attention to lower level features of the target words. This finding is compatible with the results of many recall studies that show a similar independent relation between target set size and encoding strategy (D. L. Nelson, Schreiber, \& Holley, 1992; D. L. Nelson, Schreiber, \& McEvoy, 1992-and many others reviewed in the latter source). Both sets of findings also are consistent with the hypothesis that activation of closely related associates during study is relatively automatic and does not require conscious attention to meaning. The purpose of this experiment was to determine whether target set size effects would influence knowing ratings when subjects were discouraged from attending to meaning during the testing phase. In order to discourage attention to meaning, 
the ending stems of target words were used as the test cues. On each test trial, the experimenter pronounced each ending stem aloud, and the subjects repeated it aloud before responding. If the number of meaning-related associates of a target influence knowing ratings even when subjects must attend to rhyme-based information, this finding would be consistent with the hypothesis that target set size influenced POK ratings as a result of relatively automatic processes that occur during the testing phase.

Many recall experiments indicate that target set size effects are the result of relatively automatic processes that occur during encoding and retrieval. Equivalent target set size effects are found on both implicit and explicit tests (D. L. Nelson, Canas, Bajo, \& Keelean., 1987; D. L. Nelson et al., 1988; D. L. Nelson, Schreiber, \& Holley, 1992); they do not depend on particular strategic orientations during either encoding or retrieval (D. L. Nelson \& McEvoy, 1979; D. L. Nelson, Schreiber, \& Holley, 1992); they occur when target words are presented at very rapid presentation rates of $250 \mathrm{msec}$ or less (Schreiber \& Carter, 1998); and meaning-related target set size effects can occur even when the attention of subjects is focused on sound rather than on meaning, either during encoding or during retrieval ${ }^{3}$ (D. L. Nelson \& Friedrich, 1980; D. L. Nelson, Schreiber, \& Holley, 1992). Meaning-related associates appear to be activated relatively automatically, and the question is whether POK ratings can capitalize on such automatic activation. In order to evaluate this possibility, target set size was crossed with type of encoding strategy as before, but ending sounds rather than associatively related words were used as test cues.

\section{Method}

Design and Subjects. The experimental design consisted of a $2 \times 2$ mixed model factorial, with target set size (small, large) manipulated within subjects and encoding strategy (concreteness classification, vowel naming) manipulated between subjects. Forty subjects participated in the experiment, with an equal number randomly assigned to each encoding condition.

Materials. The ending stems and corresponding target words are shown in Appendix B and were selected with the help of two normative data sets. The ending stems were selected from rhyme norms described in D. L. Nelson and McEvoy (1979). For these norms, many subjects were asked to generate the first word that came to mind that rhymed with ending sounds. Cue set size and cue-to-target strength were calculated with these norms, and the rhyming responses that also had been used in the separate association norms described in Experiments 1 and 2 formed the pool of potential target words. Using the rhyme as well as the association norms, it was possible to vary meaning-related target set size and to equate characteristics of the ending sound cues, such as preexisting cue-to-target strength and rhyme set size, for each level of target set size. For example, in the norming task involving stems, the ending sound/op/ elicited 16 rhyme-related responses, and the probability with which stop was given was .06; when stop served as a stimulus word in the associative norming task, eight different meaningfully related associates were given by the subjects in the sample. Similarly, the ending sound /ell/also elicited 16 rhyme-related responses, and the word tell also was given with a probability of .06 ; but, in contrast, 19 meaningfully related associates were given when tell served as a stimulus in the associative norming task. Using this procedure for selecting materials, two separate lists of 44 cues and targets were constructed, with an equal number of subjects randomly assigned to each list. Pooled across lists, target words with relatively small sets averaged 6.77 associates $(S D=1.26)$, and target words with larger sets averaged 19.11 associates $(S D=2.44)$. For each level of target set size, preexisting cue-to-target strength averaged 0.09 $(S D=0.08)$ and $0.09(S D=0.08)$, cue set size averaged 12.93 $(S D=6.06)$ and $13.41(S D=5.52)$, target concreteness averaged $5.24(S D=1.14)$ and $4.99(S D=1.23)$, and printed frequency of targets averaged 33.41 occurrences per million $(S D=47.88)$ and 50.54 occurrences per million $(S D=88.77$ ). None of the differences between these other attributes was significant, with all $F \mathrm{~s}$ close to unity.

Procedure. The procedure was similar to the procedure in Experiment 2. Study words were presented one at a time at a 4-sec rate, and, after reading each word aloud, the subjects classified it with respect to concreteness or named its vowels, depending on encoding condition. After study, the subjects made POK ratings and then performed a cued recall test. During both the knowing rating and recall phases, the ending stems of the studied words were presented visually on a computer screen. Despite the visual presentation, attention to the sound of the stem was emphasized. The experimenter pronounced each stem aloud, and the subjects were asked to repeat it aloud themselves before responding. Once again, the purpose of pronouncing the stems aloud before responding was to focus the subjects' attention on the phonological characteristics of the cue when making POK ratings or, in the case of recall, retrieving the studied word. RTs were not analyzed in the present study, because of the delay caused by pronouncing the ending stems for the subjects and having subjects repeat them aloud before responding.

\section{Results and Discussion}

Mean POK ratings for unrecalled and recalled items are shown in Figure 5. Mean POK ratings were higher when targets were linked to smaller associative sets (4.40) than when they were linked to larger associative sets (4.18), indicating that a target set size effect was obtained. The statistical analysis indicated that the difference between these means was significant $\left[F(1,38)=7.88, M S_{\mathrm{e}}=\right.$ 0.23 ]. Apparently, the number of meaning-related associates of a target influences knowing ratings, even when an ending stem is provided as the initiating stimulus. Mean POK ratings also were higher for targets that later were recalled (4.90), as compared with those that were not recalled (3.68), replicating the pattern obtained in the first two experiments. Also replicating the pattern in Experiment 2, targets encoded in the concreteness classification condition received higher ratings (4.46) than those encoded in the vowel-naming condition (4.11). The results of the statistical analysis indicated that the effect of correctness of the response on the eventual recall test was significant $\left[F(1,38)=107.47, M S_{\mathrm{e}}=0.55\right]$, but, unlike Experiment 2, the main effect of encoding task did not reach the criterion for significance in the present experiment $[F(1,38)=2.33, p<.13]$. The statistical analysis also indicated that the interaction between target set size and encoding condition was not even close to the criterion for significance, with $F<1$.

There were two reliable interactions. The first interaction was between encoding condition and the correctness of the response on the eventual recall test $[F(1,38)=$ 6.73 ]. Mean POK ratings for recalled items were significantly higher in the concreteness classification condi- 

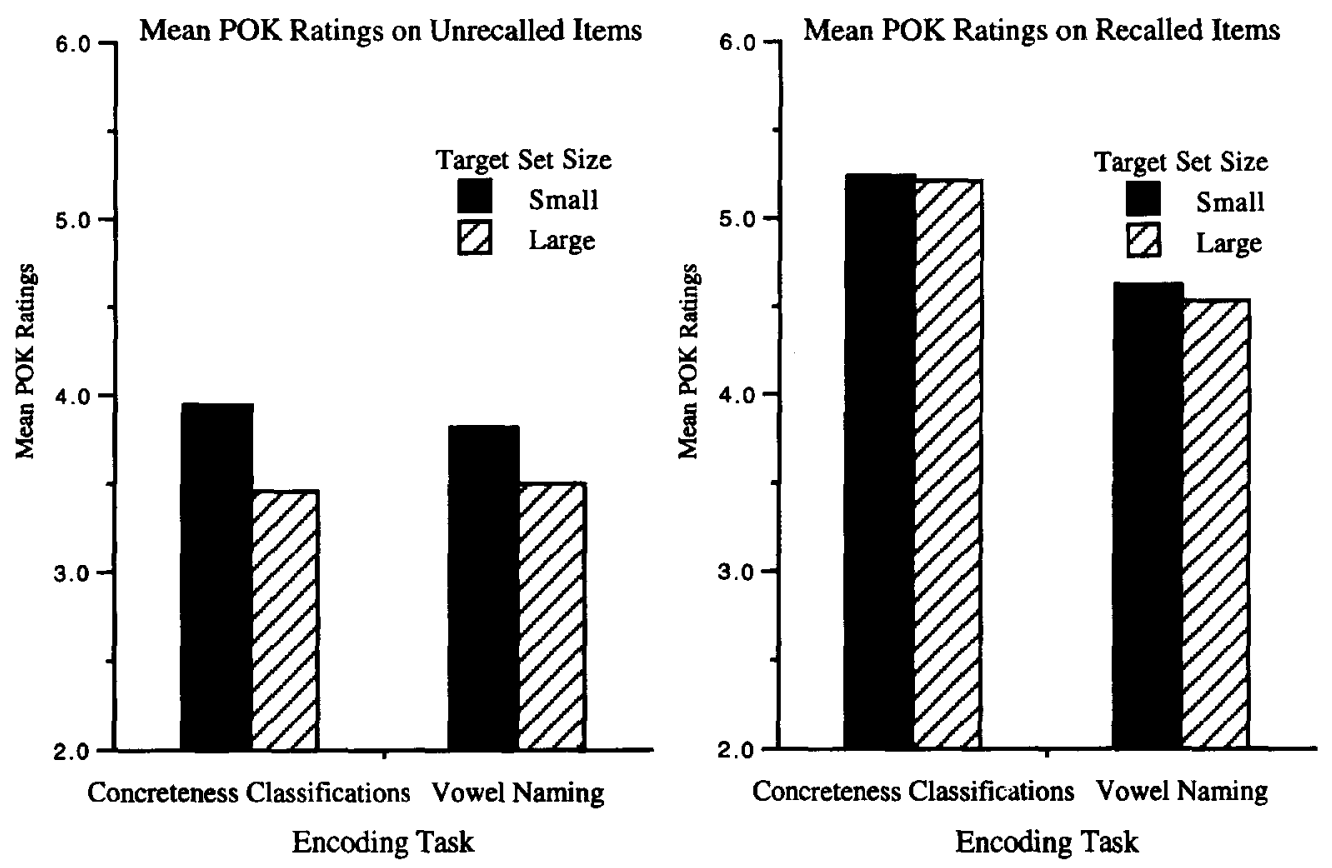

Figure 5. Mean prediction-of-knowing (POK) ratings (Experiment 3).

tion (5.22) than in the vowel-naming condition (4.57), but ratings involving unrecalled items were only slightly higher in the concreteness condition (3.71) than in the vowel-naming condition (3.65). An LSD of .33 indicated that the effect of encoding condition was significant for recalled items but not for unrecalled items. The interaction between degree of encoding and correctness also was obtained in Experiment 2 and is consistent with the hypothesis that differences between different degrees of encoding might be more easily detected for items that subjects eventually can recall, as compared with items they cannot recall.

The second reliable interaction was between target set size and correctness of the recall response $[F(1,38)=$ 6.04]. Pooled across encoding condition, mean POK ratings for unrecalled items were higher for targets linked to smaller associative sets (3.88) than for targets linked to larger associative sets (3.48). Ratings for items that subsequently were recalled were only slightly higher when targets were linked to smaller sets (4.91), as compared with targets linked to larger sets (4.88). An LSD of .21 indicated that the effect of target set size was significant for unrecalled items but not for recalled items. One interpretation of this interaction is that it occurred in the present experiment as a result of random variation. This interpretation is based on the findings of both Experiments 1 and 2 that equivalent target set size effects were obtained for unrecalled and recalled items and on similar findings involving cue set size effects (Schreiber \& D. L. Nelson, in press; Schreiber \& Sergent, 1998) in which equivalent cue set size effects were obtained for both kinds of items. A second interpretation is that, although item selection effects did not appear to be a prob- lem in Experiments 1 and 2, item selection effects could have influenced the results of the present experiment in some way. In this case, a reasonable approach would be to rely on the main effect of target set size, in which means for recalled and unrecalled items are pooled and item selection effects could not contribute to the results. The main effect of target set size for unrecalled and recalled items pooled was significant, indicating that, overall, the number of associates linked to a target influences POK ratings, especially when there are no problems associated with selection effects.

Probabilities of recall. Mean probabilities of recall are shown in the fifth and sixth rows of Table 1. Recall was more likely when targets were linked to smaller associative sets (.56) than when they were linked to larger sets (.46). The statistical analysis indicated that the difference between these means was significant $[F(1,38)=$ $19.78, M S_{\mathrm{e}}=0.01 \mathrm{]}$. Recall also was more likely after concreteness classifications (.59) than after vowel naming (.43), and the difference between these means also was significant $\left[F(1,38)=23.22, M S_{\mathrm{e}}=0.02\right]$. The interaction between target set size and encoding condition was not significant $(F<1)$. Both the finding that the number of meaning-related associates of a target influenced the likelihood of recalling the target, even when the test cue did not provide direct access to these associates, and the finding that degree of encoding influenced the likelihood of recall with ending sounds as test cues replicate previous findings (see, e.g., D. L. Nelson, Schreiber, \& Holley, 1992; D. L. Nelson, Schreiber, \& McEvoy, 1992). It is worth noting that the difference between mean recall probabilities for the concreteness classification condition and the vowel-naming condition was not as large 
in the present experiment as it was in Experiment 2. The difference between these conditions was nearly .30 in Experiment 2 , but the difference of .16 in the present experiment is much smaller, indicating that the manipulation of degree of encoding was not as effective in the present experiment. The reduced effectiveness of encoding condition in the present experiment could be related to the fact that ending stem cues were used rather than meaning-related cues. Specifically, vowel naming may be somewhat more transfer appropriate for ending stem cues than for associative meaning cues, because both vowel naming and ending stems require more attention to perceptually related information (Morris, Bransford, $\&$ Franks, 1977). The pattern of means across Experiment 2 and the present experiment is consistent with this speculation. An examination of means for comparable conditions indicates that the means were nearly identical in all conditions, except that recall was much more likely following vowel naming in the present experiment than following vowel naming in Experiment 2.

\section{GENERAL DISCUSSION}

The most important contribution of the present investigation was in showing that POK and FOK ratings were influenced by the number of associates linked directly to target words, and these findings have important implications for the cue effectiveness hypothesis. The fact that target set size effects were found at all indicates that associates of the target played a role, a result that suggests that knowing ratings are not influenced only by cue-based sources of information. In each of the three experiments, knowing ratings were higher when targets were linked to smaller sets of associates than when they were linked to larger sets of associates. This pattern is compatible with the specific hypothesis that associates linked to the target produce competition and that people can detect this competition when predicting subsequent memory performance. Target set size effects consistent with competition were obtained in each experiment under different conditions of encoding and testing. Experiments 2 and 3 only examined POK ratings, because similar results had been obtained for both POK and FOK ratings in Experiment 1 and because POK ratings permitted analyses based on recalled items, unrecalled items, or both kinds of items pooled. The most important findings involving the magnitude of POK ratings in Experiment 2 were that equivalent target set size effects were found when people classified studied words with respect to concreteness, as compared with naming their vowels. Regardless of the kind of encoding task, POK ratings were significantly higher when targets were linked to smaller sets of associates than when they were linked to larger sets of associates. The results of Experiment 3 showed further that meaning-related target set size effects can occur when ending sounds are used as the initiating stimulus and people are instructed to attend to phonological information directly activated by these sounds. Target set size effects were obtained, even when subjects were not en- couraged to attend to the meaning of target words during study (vowel-naming task) and even when the test cue was linked directly only to rhyming words, a pattern consistent with the view that meaning-related target set size effects arise as a result of relatively automatic processes that occur during study and test. These findings parallel the findings of many recall experiments that also indicate that set size effects are the result of relatively automatic processes (see, e.g., D. L. Nelson, Schreiber, \& Holley, 1992; D. L. Nelson, Schreiber, \& McEvoy, 1992).

Interesting patterns of effects also were obtained when RTs were measured. In general, these patterns paralleled the patterns found in experiments involving manipulations of cue set size (Schreiber \& Sergent, 1998). Knowing ratings always were made significantly more quickly than were responses in recall, and, in both Experiment 1 and Experiment 2, about the same amount of time was required to make knowing ratings when targets were linked to smaller sets of associates and when targets were linked to larger sets of associates. These findings contrasted with the findings involving RTs in recall. Significantly less time was required to respond in recall when targets were linked to smaller sets of associates than when targets were linked to larger sets of associates. Once again, these effects parallel the effects of cue set size and suggest that FOKs may not involve the same search processes as recall. In recall, the direct relationship between set size and RTs is compatible with the view that candidate words are first activated and then sampled. The greater the number of competing items in the activated pool, the longer it takes to sample and retrieve the target. The finding that knowing ratings did not follow this pattern indicates that target set size effects may have arisen for different reasons in this task. One possibility is that knowing ratings are sensitive to competition when items initially are activated in parallel. Because items are activated in parallel, there should be no timing difference between smaller and larger sets of activated items.

The present findings have implications for several explanations of FOK effects. Up to this point, this article has focused mainly on cue-based explanations, because the impact of the present findings for such explanations is especially clear. As such, in the ensuing discussion, specific implications for cue-based explanations will be summarized first. However, it is important to note that the present findings also offer important constraints for explanations that assume that FOKs can be based on information associated with the target trace (see, e.g., Blake, 1973; Eysenck, 1979; Hart, 1967; Koriat, 1993; Koriat \& Lieblich, 1977). The nature of these constrains will be discussed next. To preview the main conclusion: Both kinds of explanations, cue- as well as target-based, appear to have limitations in terms of their ability to explain the effects of target set size. Some ways these explanations could be modified will be discussed.

Hypotheses assuming that FOKs are influenced primarily by information linked directly to the test cue seem to have clear difficulties in explaining the present data involving target set size effects. I have used the term cue 
effectiveness hypothesis throughout this article to refer to this assumption. In the present context, this hypothesis has two independent components. The first component assumes that memory predictions are influenced by the degree to which the test cue seems familiar and can be recognized (see, e.g., Metcalfe, 1993; Reder, 1987, 1988; Reder \& Ritter, 1992; Schwartz, 1994; Schwartz \& Metcalfe, 1992). The second component assumes that FOKs also can be sensitive to the amount of competition that arises when the encoding of the test cue causes directly connected associates to become activated (Schreiber \& D. L. Nelson, in press). The common denominator for these two components is the assumption that FOKs primarily are influenced by factors associated with the test cue rather than by information linked directly to the target. The main weakness of the cue effectiveness explanation, and of any explanation that assumes that FOKs are sensitive only to cue-based sources of information, is the inability to explain how target-based sources can have significant influences on FOKs. Specifically, cue-based hypotheses cannot account for the present findings, which indicate that competition produced by associates of the target can influence FOKs. This problem is especially salient for models that assume that FOKs are influenced only by a cue recognition process (see, e.g., Metcalfe, 1993; Reder, 1987, 1988; Reder \& Ritter, 1992; Schwartz \& Metcalfe, 1992). For example, in CHARM (Metcalfe, 1993) and other models (Reder, 1987, 1988; Reder \& Ritter, 1992), FOKs are determined exclusively by a cue recognition process, and, therefore, these models clearly would appear to have difficulty explaining the effects of target set size.

There are four main reasons why a cue recognition process is unlikely to be successful in explaining the effects of target set size. The first reason is that many experiments have shown that set size does not influence the probability of recognizing a previously studied word (see, e.g., Canas \& D. L. Nelson, 1986; D. L. Nelson, Canas, \& Bajo, 1987; D. L. Nelson, Canas, Casanueva, \& Castano, 1985; D. L. Nelson et al., 1988). The second reason is that associative set size is relatively independent of other attributes of words that influence recognition, such as word concreteness and word frequency (D. L. Nelson \& Schreiber, 1992; D. L. Nelson \& Xu, 1995). Thus, set size does not influence recognition, and it has effects that are independent of factors that are known to influence recognition and that could influence familiarity. A third reason for a concept other than cue recognition being necessary to explain the set size findings is based on the procedure that was used in the present experiments. Subjects had the same amount of experience with cues prior to the test phases in all of the experiments in the present series, regardless of set size. This procedure was used in order to eliminate the possibility of confounding the effects of set size with factors that may contribute to cue recognition. The associatively related cues in Experiments 1 and 2 were presented only during the test phase and not during study, ensuring that the subjects had the same amount of experience with these cues, regardless of set size. Ending stems differ from associative cues, because the ending sounds of target words obviously are present during study. However, the amount of experience with the endings of target words during study was equivalent for items that differed in terms of set size. Thus, the effects of set size were independent of factors that are known to influence cue recognition even with ending cues.

The fourth and final reason that a concept other than cue recognition seems necessary for explaining the present findings is that the effects of target set size concern information that is associated with the representation of the target. Therefore, it is difficult to understand how the effects of target set size could be explained by the concept $c u e$ recognition. It is especially difficult to imagine how cue recognition could explain meaning-related target set size effects when ending stems are used as the initiating stimulus. The information represented in ending stems provides direct access only to words related to the stems in terms of sound. They do not provide direct access to the meaning-related associates of the target. Thus, it is particularly unlikely that target set size effects obtained with ending stems could be attributed to cue familiarity, because the associates of a target do not even concern the same domain of information as ending stems.

The present data do not suggest that cue-based sources are unimportant but rather that FOKs can be based on target-based sources as well. That is the chief point. Another important point is that modification of the cue effectiveness hypothesis or related hypotheses, such as the cue recognition hypothesis, could be relatively straightforward, because target-based effects and cue-based effects need not be viewed as mutually exclusive. The modification of existing hypotheses primarily would require the addition of mechanisms designed to explain target set size effects rather than the deletion of existing mechanisms. It is important to underscore this point. Clearly, cue-based sources are important for FOKs, but the present investigation shows that these sources are not the only important ones.

The present findings also have implications for other models of FOK effects, particularly those that assume that FOKs can be based on sources of information directly tied to the representation of the target. The earliest model of this type was proposed by Hart (1967) and is based on strength theory, which was the dominant theory of memory when the standard FOK procedure was introduced. Although strength theory long ago was rejected as a general theory of memory, it continues to be discussed by researchers interested in FOK effects (see, e.g., Koriat, 1993; T. O. Nelson et al., 1984; Schwartz, 1994; Schwartz \& Metcalfe, 1992). For this reason, it is useful to discuss its relevance for the present research. According to a strength theory explanation, both FOKs and recall are sensitive to the strength of the target trace. Trace strength, in turn, is determined by such factors as the degree to which the target item was encoded. Cued recall is thought to have a higher threshold for trace strength than do FOKs, and this assumption can be used 
easily to explain why people can predict performance for items that they cannot recall. Memory predictions presumably are more sensitive to trace strength than is cued recall.

The only obvious way to apply strength theory to the present findings is to add the assumption that target set size influences the strength of the target trace. It would have to be assumed that, with fewer activated associates, the strength of the target trace is higher than it is with a greater number of activated associates. As such, on average, knowing ratings should be higher when the target is linked to fewer associates than when it is linked to a greater number of associates. This hypothesis is synonymous with an encoding weakness explanation of target set size effects. Given a fixed total amount of activation to begin with, more activation presumably remains with the target when there are fewer related items to which activation spreads. Although this strength theory/encoding weakness explanation is compatible with the finding that FOK and POK ratings always were higher for targets linked to smaller sets, it is incompatible with other wellestablished findings, as well as with the pattern of the RTs that were obtained. One important problem is that this explanation predicts that target set size should affect performance on all memory tests, because the trace for targets linked to smaller sets always should be stronger. This assumption is incompatible with the existing literature showing that target set size does not affect performance on all tests. For example, target set size does not influence recognition performance. Once again, despite many attempts to find preexisting set size effects in recognition tasks under a variety of conditions, such effects have not been found in standard single-item or forced-choice tests (Canas \& D. L. Nelson, 1986; D. L. Nelson, Canas, \& Bajo, 1987; D. L. Nelson, Canas, et al., 1985; D. L. Nelson et al., 1988; see also D. L. Nelson, Schreiber, \& McEvoy, 1992, for a discussion of other tests which are uninfluenced by target set size).

Another problem for the strength theory/encoding weakness explanation is that it is difficult to understand how it could explain the pattern of the RTs that were obtained in the present series. Why does target set size have no effect on rating latencies but substantial effects on recall latencies? If knowing ratings and recall both were based on the single dimension of trace strength, the same pattern of RTs should have been obtained for both tasks. RTs for both knowing ratings and recall should have been faster for targets linked to smaller associative sets, because the underlying memory trace should always be stronger and memory access times quicker than those for targets linked to larger associative sets. The challenge for strength theory or any explanation of the present findings would be to incorporate assumptions that address not only the pattern of FOK and POK ratings that were obtained but also the pattern of the RTs that were obtained. In addition, the presence and absence of set size effects on other memory tests must also be taken into account, because all of these factors considered simultaneously provide valuable constraints on theorizing.
The partial-retrieval hypothesis is a second type of explanation assuming that FOKs could be influenced by target-based information (see, e.g., Blake, 1973; Eysenck, 1979; Koriat, 1993; Koriat \& Lieblich, 1977). According to this hypothesis, people compute FOKs by searching memory for the target and then noting how much related information enters consciousness during the course of search. Such information could include candidate answers or neighboring targets that are retrieved, as well as lexical and semantic fragments of the target that are retrieved. In general, the more related information that comes to mind during the course of search, the higher the FOK. This assumption is based partly on investigations of TOT experiences. When people have a TOT experience, they typically have a high FOK, and they sometimes can report linguistic attributes of the target word, such as the beginning letter, the number of syllables, or words that are related in meaning or sound (for a review, see Brown, 1991). Presumably, when such information comes to mind during the course of searching for the target, it serves as positive evidence that some information about the target is known and gives rise to a feeling that the target could be retrieved in the future, even though it might not be retrievable at the moment. In contrast to other versions of this hypothesis, Koriat's (1993) application of the partial-retrieval principle assumes that the information that comes to mind during search need not be linked to the target but could be any kind of information, whether it is linked to the target or not.

Regardless of whether Koriat's (1993) modification of the standard partial-retrieval principle is taken into account, one problem with this principle in general concerns the issue of cause and effect. The question is whether FOKs are caused by conscious access to related information or whether they are computed on some other basis and whether, when they happen to be more positive, a person is more willing to report related information. When given a higher FOK, a person may be more likely to volunteer related information than when given a lower FOK. The existing literature cannot be used to determine the true direction of this cause and effect relationship (see also Metcalfe et al., 1993, for similar arguments).

Regardless of the cause and effect question, the present findings have additional implications for the partialretrieval hypothesis. In general, the findings clearly are compatible with one very important assumption of the partial-retrieval hypothesis, but, equally clearly, they appear to be incompatible with several others. The finding that target set size effects were obtained at all is compatible with the position that information linked directly to the target can influence knowing judgments even when the target itself cannot be recalled. However, there are three main assumptions of the partial-retrieval hypothesis that are incompatible with the present findings. One of the central assumptions of this hypothesis is that FOKs should be more positive, the more related information is accessed in memory. This assumption seems to predict that knowing ratings should have been lower for target words linked to smaller associative sets than for those 
linked to larger sets. This is opposite to the pattern that was obtained in all three reported experiments, because higher ratings always were obtained when targets were linked to smaller sets. Assuming a direct relationship between knowing ratings and the number of related items accessed also is incompatible with the cue set size findings (Schreiber \& D. L. Nelson, in press). These findings indicated that knowing ratings for cues linked to smaller associative sets always were higher than those for cues linked to larger sets, and this also is opposite to the pattern that seems to be predicted by the partial-retrieval hypothesis.

A second assumption of the partial-retrieval hypothesis that clearly seems to be incompatible with the present findings is that FOKs always are based on executing a search of memory, as presumably must occur in cued recall. This assumption predicts that RTs when making knowing ratings should follow the same pattern as RTs in recall. For both tasks- knowing ratings and recallRTs always should be faster when the target is linked to fewer items, because there is less related information that must be searched as attempts are made to retrieve the target. Once again, this pattern did not occur in any of the present experiments or in those investigating cue set size effects (Schreiber \& Sergent, 1998). In the present series, target set size only influenced RTs in recall and had no affect on RTs in either the FOK or the POK tasks. Apparently, knowing ratings are not always based on searching memory, as in recall.

The third and final assumption of the partial-retrieval hypothesis that seems to be at odds with the present findings concerns the issue of conscious access to related information. The hypothesis assumes that neighboring items could influence FOKs, provided they are retrieved explicitly, as when a person has a TOT experience. When more items can be brought to mind, FOKs should be higher than they are when fewer items can be brought to mind. The reasons why the present data are at odds with this assumption can be understood most clearly by considering the results of Experiment 3, in which meaningrelated target set size effects were obtained even when ending stems were used as the initiating stimulus. These stems encouraged conscious attention to word sound rather than to meaning. Chances are that any information that a person might retrieve consciously would be restricted to the phonological domain; yet meaning-related target set size effects still were obtained. Such effects were obtained even when people could not recall the target and even when they had encoded the targets during the learning phase by naming their vowels. This finding indicates that meaning-related target set size can influence knowing ratings, even when conscious attention to meaning is severely curtailed both during the encoding and during the test trial. These findings, as well as many others obtained in recall studies (reviewed in D. L. Nelson, Schreiber, \& McEvoy, 1992), suggest that target set size effects may be more closely associated with relatively automatic memory access processes than with the conscious and deliberate retrieval of related information.
Without making a distinction between what is retrieved consciously and deliberately and what is activated relatively automatically and implicitly, the finding that set size had any affect at all on FOKs conflicts with the partial-retrieval hypothesis. As such, an important modification of the partial-retrieval hypothesis, which would place it in a better position to explain the effects of target set size, would be to loosen the restriction that knowing ratings only can be influenced by neighboring items that can be retrieved explicitly. However, even if this general modification were made, the partial-retrieval hypothesis would still need to add other, more specific mechanisms that address the other issues that were raised. What is needed is a way to explain why knowing ratings are higher for targets linked to smaller sets of associates than for targets linked to larger sets of associates under different conditions of encoding and testing and why target set size has no effect on knowing rating latencies but substantial effects on recall latencies.

One way to modify existing hypotheses, such as the cue effectiveness hypothesis and the partial-retrieval hypothesis, would be to incorporate assumptions similar to those included in the present adaptation of the PIER model (D. L. Nelson, Schreiber, \& Holley, 1992; D. L. Nelson, Schreiber, \& McEvoy, 1992). Although this model was not designed to explain FOK effects, it nevertheless was designed to explain both cue and target set size effects, and its main strength lies in specifying what kind of information is activated during the study and test trial in the associative cuing task. To explain cue set size effects, PIER assumes that the test cue activates connections to closely related neighboring concepts relatively automatically and in parallel. The activation of these connections increases the accessibility of related concepts, but it also produces competition in the form of background noise, in the same way that background noise exists in a room filled with many people. Both kinds of noise compete with the signal that represents activation of the target. Cue set size influences FOKs because, when the cue activates fewer connections to neighboring concepts, there is less noise than there is when the cue activates a larger number of connections. When there is less noise, there is less interference with the amount of activation received by the target. When making knowing ratings, people apparently can detect the amount of noise, so ratings are higher when the cue activates fewer connections than when it activates more connections to competing concepts.

Target set size influences FOKs because, when the test cue activates the connection to the target, its representation can reactivate connections to neighboring concepts that were originally activated during study. Even though activation begins with the test cue, it spreads from this source, eventually activating associates of the target. Once the connections to associates of the target have been reactivated, they contribute to the amount of competition, or noise, that is produced. The more connections that are reactivated, the greater the amount of competition. FOKs are influenced by target set size because they are sensitive to the amount of competition. An additional assump- 
tion is that the activation of connections to neighboring concepts during study is relatively automatic. Activation is automatic, in the sense that these connections are activated regardless of whether targets are analyzed explicitly in terms of meaning or whether they are analyzed in terms of lower level perceptual characteristics. The reactivation of connections during testing also is relatively automatic. Connections to meaning-related associates of the target can become reactivated, even when attention is focused on the perceptual characteristics of the test cue, such as its sound (D. L. Nelson, Schreiber, \& McEvoy, 1992).

Meaning-related target set size effects occur when an ending stem is presented as a cue during testing and attention is focused on rhyme. The ending stem causes the phonological representation of the target to become activated. Activation can spread from this representation to the target's semantic representation. The semantic representation, in turn, becomes the source of further activation. Specifically, the semantic representation can reactivate connections to meaning-related associates of the target that were originally activated during study. Once these connections are reactivated, they can contribute to the amount of competition, even though the original initiating stimulus only provided direct access to phonologically related information. During this activation process, subjects primarily have conscious access to the initiating stimulus, the instructions guiding its use, and the end product of the activation process. The end product includes a sense of the amount of competition, or background noise, produced when activation is received by connections to neighboring concepts, and this activation can interfere with the amount of activation received by the target. It is this end product that presumably contributes to a sense of FOK.

This explanation can account for the effects of cue set size and target set size on the magnitude of FOKs with different types of cues and encoding instructions. Additional assumptions can be used to explain the interactive pattern between set size and type of test when RTs are measured. PIER assumes that activation is in parallel and is very fast, whereas search represents a much slower, serial process. When faced with the task of retrieving a specific item from the pool of items that are activated, search processes are required. Because recall requires the retrieval of a specific item, search processes must be initiated. RTs using search are faster when fewer concepts are activated than when more concepts are activated, because fewer concepts must be sampled when attempting to retrieve the target. Making an FOK rating does not require the retrieval of a specific item. Rather, to make an FOK rating, subjects are required to sense their FOKs. The rapid activation of connections to competing concepts is sufficient to produce a sense of the amount of competition, or background noise, and this activation process is sufficient to contribute to a sense of FOK. Because activation is rapid and occurs prior to search, the amount of time needed to make a rating is less than the amount of time needed to respond in recall. In addition, because activation is in parallel, there is no timing difference for FOKs when fewer connections to competing concepts are activated, as compared with more connections being activated.

The explanation of set size effects based on the activation of connections to competing concepts essentially consists of automatically computing a kind of signal-tonoise ratio (Schreiber, 1993; Schreiber \& D. L. Nelson, in press; Schreiber \& Sergent, 1998). The signal component represents the amount of activation received by the target and may be affected by the degree to which the target is encoded. The present findings indicated that this kind of information influenced FOKs especially when items were strong enough to recall. Other findings show that FOKs are influenced by the degree to which the cue is encoded (see, e.g., Schwartz \& Metcalfe, 1992). These findings indicate that the signal component may be influenced by the amount of activation received by the representation of the test cue. In contrast, the noise component represents the amount of activation received by connections to concepts that compete with the target. It is the sensitivity to this component that produces effects of cue set size and effects of target set size.

Although these assumptions, based on a modification of PIER, are sufficient to explain the present findings, other models making different representational assumptions may find other ways that also could account for the data. CHARM (Metcalfe, 1993) is a single-layer connectionist model that uses quite different representational assumptions than does the associative network approach in PIER. The challenge for this model and for others would be to add assumptions concerning the role of a target's associates. The challenge for models such as PIER, on the other hand, is to find ways to address the functional role that memory predictions play in memory. The present data do not allow a firm conclusion, but they do provide some guidance. The present findings are quite compatible with the view that FOKs could play a role in the early stages of retrieval, because both FOK and POK ratings always were made much more quickly than was recall, and the effects of target set size on RTs suggested they were more likely associated with parallel activation processes rather than with search. Because activation occurs prior to search, FOKs very well could be used as a basis for making search initiation decisions or for regulating other processing during the early stages of retrieval (see, e.g., Metcalfe, 1993; Reder, 1988; Schreiber, 1993; Schreiber \& D. L. Nelson, in press; Schreiber \& Sergent, 1998).

\section{REFERENCES}

ANDERSON, J. R. (1983). A spreading activation theory of memory. Journal of Verbal Learning \& Verbal Behavior, 22, 261-295.

BLAKE, M. (1973). Prediction of recognition when recall fails: Exploring the feeling of knowing phenomenon. Journal of Verbal Learning \& Verbal Behavior, 6, 89-94.

Brown, A. S. (1991). A review of the tip-of-the-tongue experience. Psychological Bulletin, 109, 204-223.

Canas, J., \& Nelson, D. L. (1986). Recognition and environmental context: The effect of testing by phone. Bulletin of the Psychonomic Society, 24, 407-409.

Collins, A. M., \& LofTus, E. F. (1975). A spreading activation theory of semantic processing. Psychological Review, 82, 407-428. 
Craik, F. I. M., \& Tulving, E. (1975). Depth of processing and the retention of words in episodic memory. Journal of Experimental Psychology: General, 104, 268-294.

EYSENCK, M. W. (1979). The feeling of knowing a word's meaning. British Journal of Psychology, 70, 243-251.

Gillund, G., \& Shiffrin, R. M. (1984). A retrieval model for both recognition and recall. Psychological Review, 91, 1-67.

HART, D. (1967). Memory and the memory-monitoring process. Journal of Verbal Learning \& Verbal Behavior, 6, 685-691.

KINTSCH, W. (1988). The role of knowledge in discourse comprehension A construction-integration model. Psychological Review, 95, 163-182.

KorIat, A. (1993). How do we know that we know? The accessibility model of the feeling of knowing. Psychological Review, 100, 609-639

Koriat, A., \& Lieblich, I. (1977). A study of memory pointers. Acta Psychologica, 41, 151-164.

KUČERA, H., \& FRANCIS, W. N. (1967). Computational analysis of present day American-English. RI: Brown University Press.

LEONESIO, R. J., \& NELSON, T. O. (1990). Do different metamemory judgments tap the same underlying aspects of memory? Journal of Experimental Psychology: Learning, Memory, \& Cognition, 16, 464-470.

McEvoY, C. L., \& Holley, P. (1990). Aging and the stability of activation and sampling in cued recall. Psychology \& Aging, 5, 589-596.

MetCALFE, J. (1993). Novelty monitoring, metacognition, and control in a composite holographic associative recall model: Implications for Korsakoff amnesia. Psychological Review, 99, 1-20.

Metcalfe, J., Schwartz, B. L., \& Joaquim, S. G. (1993). The cuefamiliarity heuristic in metacognition. Journal of Experimental Psychology: Learning, Memory, \& Cognition, 19, 851-864.

MorRis, C. D., BRANSFORD, J. D., \& FranKs, J. J. (1977). Levels of processing versus transfer appropriate processing. Journal of Verbal Learning \& Verbal Behavior, 16, 519-533.

NELSON, D. L. (1989). Implicitly activated knowledge and memory. In C. Izawa (Ed.), Current issues in cognitive psychology: The Tulane Flow eree Symposium on Cognition (pp. 129-162). Hillsdale, NJ: Erlbaum.

Nelson, D. L., \& Bajo, M.-T. (1985). Prior knowledge and cued recall: Category size and dominance. American Journal of Psychology, 98 503-517.

Nelson, D. L., Bajo, M.-T., \& Canas, J. (1987). Prior knowledge and memory: The episodic encoding of implicitly activated associates and rhymes. Journal of Experimental Psychology: Learning, Memory, \& Cognition, 13, 54-63.

Nelson, D. L., Bajo, M.-T., \& Casanueva, D. (1985). Prior knowledge and memory: The influence of category size as a function of intention and distraction. Journal of Experimental Psychology: Learning, Memory, \& Cognition, 11, 94-105.

Nelson, D. L., Bennett, D. J., Gee, N. R., Schreiber, T. A., \& MCKINNEY, V. M. (1993). Implicit memory: Effects of network size and interconnectivity on cued recall. Journal of Experimental Psychology: Learning, Memory, \& Cognition, 19, 747-764.

Nelson, D. L., CANAS, J., \& BAJo, M.-T. (1987). The effects of natural category size on memory for episodic encodings. Memory \& Cognition, 15, 133-140.

Nelson, D. L., Canas, J., Bajo, M.-T., \& Keelean, P. (1987). Comparing word fragment completion and cued recall with letter cues. Journal of Experimental Psychology: Learning, Memory, \& Cognition, 13, 542-552.

Nelson, D. L., Canas, J., Casanueva, D., \& Castano, D. (1985). Prio knowledge and recognition. Journal of Experimental Psychology: Learning, Memory, \& Cognition, 98, 379-397.

NeLSON, D. L., \& Friedrich, M. A. (1980). Encoding and cuing sounds and senses. Journal of Experimental Psychology: Learning, Memory, \& Cognition, 6, 717-731.

Nelson, D. L., Gee, N, R., \& Schreiber, T. A. (1992). Sentence encoding and implicitly activated memories. Memory \& Cognition, 20 , 643-654.

NeLson, D. L., \& McEvoy, C. L. (1979). Encoding context and set size. Journal of Experimental Psychology: Human Learning \& Memory, 5, 292-314

Nelson, D. L., MCEvoy, C. L., \& BaJo, M.-T. (1988). Lexical and semantic search in cued recall, fragment completion, perceptual identification, and recognition. American Journal of Psychology, 101, 465-480.
Nelson, D. L., McEvoy, C. L., \& Friedrich, M. A. (1982). Extralist cuing and retrieval inhibition. Journal of Experimental Psychology: Learning, Memory, \& Cognition, 8, 89-105.

Nelson, D. L., McEvoy, C. L., \& Schreiber, T. A. (1995). The University of South Florida word association, rhyme, and word fragment norms. Unpublished manuscript.

NELSON, D. L., \& SCHREIBER, T. A. (1992). Word concreteness and word structure as independent determinants of recall. Journal of Memory \& Language, 31, 237-260.

Nelson, D. L., Schrerber, T. A., \& Holley, P. E. (1992). The retrieval of controlled and automatic aspects of meaning on direct and indirect tests. Memory \& Cognition, 20, 671-684.

Nelson, D. L., Schreiber, T. A., \& McEvoy, C. L. (1992). Processing implicit and explicit representations. Psychological Review, 99, 322-348.

NeLSON, D. L., \& Xu, J. (1995). Effects of implicit memory on explicit recall: Set size and word-frequency effects. Psychological Research, 57, 203-214

Nelson, T. O., Gerler, D., \& Narens, L. (1984). Accuracy of feelingof-knowing judgments for predicting perceptual identification and relearning. Journal of Experimental Psychology: General, 113, 282-300.

Nelson, T. O., Leonesio, R. J., Shimamura, A. P., Landwehr, R. S., \& NARENS, L. (1982). Overlearning and the feeling of knowing. Journal of Experimental Psychology: Learning, Memory, \& Cognition, 8, 279-288.

NelsoN, T. O., \& NARENS, L. (1990). Metamemory: A theoretical framework and new findings. In G. Bower (Ed.), The psychology of learning and motivation (Vol. 26, pp. 125-141). San Diego, CA: Academic Press.

RaAiJmakers, J. G. W., \& Shiffrin, A. M. (1981). Search of associative memory. Psychological Review, 88, 93-134.

REDER, L. M. (1987). Strategy selection in question answering. Cognitive Psychology, 19, 90-138.

REDER, L. M. (1988). Strategic control of retrieval strategies. In G. Bower (Ed.), The psychology of learning and motivation (Vol. 22, pp. 227259). New York: Academic Press.

REDER, L. M., \& RitTER, F. E. (1992). What determines initial feeling of knowing? Familiarity with question terms, not with the answer. Journal of Experimental Psychology: Learning, Memory, \& Cognition, 18, 435-451.

SCHACTER, D. L. (1983). Feeling of knowing in episodic memory. Journal of Experimental Psychology: Learning, Memory, \& Cognition, 9, 39-54. SCHREIBER, T. A. (1993). Feelings of knowing are based upon implicitly activated knowledge about the test cue and the target. Unpublished doctoral dissertation, University of South Florida.

SCHREIBER, T. A., \& CARTER, K. (1998). The activation and suppression of preexisting associations in an episodic memory task. Manuscript submitted for publication.

Schreiber, T. A., \& Nelson, D. L. (in press). The relation between feelings of knowing and the number of neighboring concepts linked to the test cue. Memory \& Cognition.

SChreIBer, T. A., \& SERGENT, S. D. (1998). Dissociative effects of cue set size on predictions of knowing and actual knowing: Further evidence for the activation-competition hypothesis. Manuscript submitted for publication.

SCHWARTZ, B. L. (1994). Sources of information in metamemory: Judgments of learning and feelings of knowing. Psychonomic Bulletin \& Review, 1, 357-375.

Schwartz, B. L., \& MetCalfe, J. (1992). Cue familiarity but not target retrievability enhances feeling-of-knowing judgments. Journal of Experimental Psychology: Learning, Memory, \& Cognition, 18, 1074-1083.

TABACHNIK, B. G., \& FidELL, L. S. (1983). Using multivariate statistics. New York: Harper \& Row.

\section{NOTES}

1. Processing Implicit and Explicit Representations.

2. A spotlight metaphor can be used to illustrate the difference between competition that arises when items are activated and competition that arises during sampling. Assume that activation is similar to a series of spotlights being turned on in parallel and that these point to items in long-term memory. Without examining what is at the end of each spotlight, it is possible to detect that either few or many competing items are 
being pointed to, because, with many spotlights, there is less focus. However, it should not take any less time to detect competition with few as compared with many spotlights, because they are turned on in parallel. A process analogous to sampling would be necessary to examine what is at the end of each spotlight, and this type of process presumably must be used in recall. In this case, less time would be required, on average, to examine what is at the end of each spotlight.
3. Even though equivalent target set size effects are found for these conditions, they easily can be eliminated or even reversed in certain circumstances (see D. L. Nelson, Schreiber, \& McEvoy, 1992), and they occur only for studied target items rather than for unstudied items, indicating that some encoding of the target word is necessary in order to obtain target set size effects (see, e.g., D. L. Nelson, Schreiber, \& Holley, 1992; Schreiber \& Carter, 1998; Schreiber \& Sergent, 1998).

APPENDIX A

Materials for Experiment 2

\begin{tabular}{|c|c|c|c|c|c|c|c|}
\hline Cues & Targets-Small & Cues & Targets-Large & Cues & Targets-Small & Cues & Targets-Large \\
\hline \multicolumn{4}{|c|}{ List 1} & \multicolumn{4}{|c|}{ List 2} \\
\hline bee & hive & crawl & baby & cow & calf & like & admire \\
\hline night & dark & mud & dirt & task & job & pizza & cheese \\
\hline joke & laugh & slippers & robe & jump & hop & vote & elect \\
\hline thimble & needle & window & door & prince & king & shoot & gun \\
\hline circle & round & $\log$ & fire & blade & razor & bribe & money \\
\hline sign & stop & elk & moose & grief & $\mathrm{sad}$ & throat & neck \\
\hline test & exam & storm & rain & beach & sand & pillow & soft \\
\hline horse & pony & satin & silk & sticky & glue & thaw & melt \\
\hline eye & sight & string & rope & boat & sail & crisp & crunch \\
\hline bristle & brush & sack & bag & branch & twig & roost & chicken \\
\hline foil & aluminum & meadow & flower & wedding & bride & laundry & clothes \\
\hline palace & king & wave & ocean & wasp & bee & link & connect \\
\hline butter & margarine & poker & card & burst & bubble & ivory & elephant \\
\hline tavern & pub & socket & plug & mistake & error & pear & fruit \\
\hline exit & enter & liberty & freedom & drip & faucet & helmet & motorcycle \\
\hline fix & repair & raisin & grape & knuckle & fist & ceramic & pottery \\
\hline hen & rooster & wed & marriage & wit & humor & decay & rot \\
\hline warrior & soldier & mob & gang & seasoning & salt & liberty & statue \\
\hline season & winter & pasta & Italian & vision & sight & path & trail \\
\hline cousin & aunt & reptile & lizard & start & stop & barrier & wall \\
\hline
\end{tabular}

APPENDIX B

Materials for Experiment 3

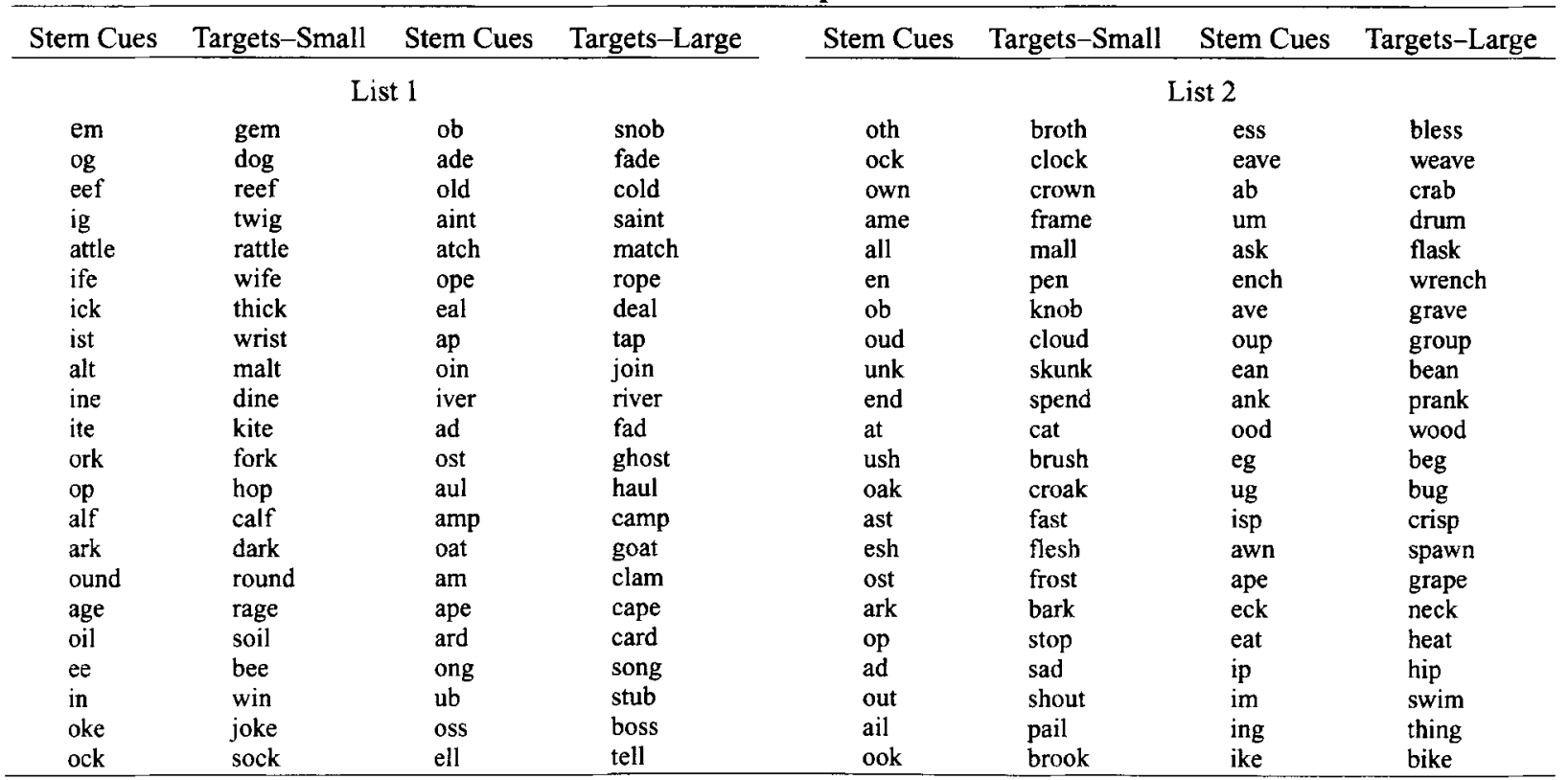

